

\title{
Uniformly rigid spaces
}

\author{
Christian Kappen
}

\begin{abstract}
We define a new category of nonarchimedean analytic spaces over a complete discretely valued field, which we call uniformly rigid. It extends the category of rigid spaces, and it can be described in terms of bounded functions on products of open and closed polydiscs. We relate uniformly rigid spaces to their associated classical rigid spaces, and we transfer various constructions and results from rigid geometry to the uniformly rigid setting. In particular, we prove an analog of Kiehl's patching theorem for coherent ideals, and we define the uniformly rigid generic fiber of a formal scheme of formally finite type. This uniformly rigid generic fiber is more intimately linked to its model than the classical rigid generic fiber obtained via Berthelot's construction.
\end{abstract}

1. Introduction

2. Uniformly rigid spaces 346

3. Coherent modules on uniformly rigid spaces 375

4. Comparison with the theories of Berkovich and Huber 383

Acknowledgements 386

References $\quad 386$

\section{Introduction}

Let $K$ be a nonarchimedean field, and let $R$ be its valuation ring, equipped with the valuation topology. Grothendieck had suggested that rigid spaces over $K$ should be viewed as generic fibers of formal schemes of topologically finite (tf) type over $R$, that is, of formal schemes which are locally isomorphic to formal spectra of quotients of strictly convergent power series rings in finitely many variables

$$
R\left\langle T_{1}, \ldots, T_{n}\right\rangle .
$$

He envisaged that rigid spaces should, in a suitable sense, be obtained from these formal schemes by tensoring over $R$ with $K$. In accordance with this point of view,

MSC2010: primary 14G22; secondary 14K15.

Keywords: semiaffinoid, uniformly rigid, formally finite type, rigid geometry, formal geometry,

Berthelot construction. 
there is a generic fiber functor

$$
\text { rig : }\left(\begin{array}{c}
\text { formal } R \text {-schemes } \\
\text { of locally tf type }
\end{array}\right) \rightarrow(\text { rigid } K \text {-spaces })
$$

characterized by the property that it maps affine objects to affinoid spaces such that, on the level of functions, it corresponds to the extension of scalars functor - $\otimes_{R} K$. This functor was more closely studied first by Raynaud and later by Bosch and Lütkebohmert; they proved that it induces an equivalence between the category of quasiparacompact and quasiseparated rigid $K$-spaces and the category of quasiparacompact admissible formal $R$-schemes, localized with respect to the class of admissible blowups [Raynaud 1974; Bosch and Lütkebohmert 1993a; Bosch 2005, Theorem 2.8/3].

From now on, let us assume that the absolute value on $K$ is discrete, so that $R$ is noetherian. Berthelot has extended the generic fiber functor to the class of formal $R$-schemes of locally formally finite (ff) type, which are locally isomorphic to formal spectra of topological quotients of mixed formal power series rings in finitely many variables

$$
R \llbracket S_{1}, \ldots, S_{m} \rrbracket\left\langle T_{1}, \ldots, T_{n}\right\rangle,
$$

where an ideal of definition is generated by the maximal ideal of $R$ and by the $S_{i}$; see [Rapoport and Zink 1996, Section 5.5; Berthelot 1996, 0.2; de Jong 1995, 7.1-7.2]. This extension of rig is characterized by the property that it maps admissible blowups to isomorphisms, where a blowup is called admissible if it is defined by an ideal that locally contains a power of a uniformizer of $R$; see [Temkin 2008, 2.1]. The extended rig functor no longer maps affine formal schemes to affinoid spaces; for example, the generic fiber of the affine formal $R$-scheme $\operatorname{Spf} R \llbracket S \rrbracket$ is the open rigid unit disc over $K$, which is not quasicompact.

While Raynaud's generic fiber functor is precisely described in terms of admissible blowups, Berthelot's extended generic fiber functor is less accessible: for example, let us consider an unbounded function $f$ on the open rigid unit disc $\mathbb{D}_{K}^{1}$. The resulting morphism $\varphi$ from $\mathbb{D}_{K}^{1}$ to the rigid projective line does not extend to models of ff type; indeed, the domain of a model of $\varphi$ cannot be quasicompact, for otherwise $f$ would be bounded. In particular, there exists no admissible blowup of Spf $R \llbracket S \rrbracket$ admitting an extension of $\varphi$, and the schematic closure of the graph of $\varphi$ in the fibered product of $\operatorname{Spf} R \llbracket S \rrbracket$ and $\mathbb{P}_{R}^{1}$ over $\operatorname{Spf} R$ does not exist. This phenomenon presents a serious obstacle if one tries for example to develop a theory of Néron models of ff type.

The main object of this article is to present a new category of nonarchimedean analytic spaces, the category of uniformly rigid spaces, which are better adapted to formal schemes of locally ff type than Tate's rigid analytic spaces. Intuitively 
speaking, uniformly rigid spaces and their morphisms are described in terms of bounded functions on finite products of open and closed unit discs. Like rigid $K$ spaces, uniformly rigid $K$-spaces are locally ringed G-topological $K$-spaces, where the letter $\mathrm{G}$ indicates that the underlying set of physical points is not equipped with a topology, but with a Grothendieck topology. Let us give a brief overview of our definitions and results.

We say that a $K$-algebra is semiaffinoid if it is obtained from an $R$-algebra of ff type via the extension of scalars functor $\cdot \otimes_{R} K$. In other words, semiaffinoid $K$-algebras are quotients of $K$-algebras of the form

$$
\left(R \llbracket S_{1}, \ldots, S_{m} \rrbracket\left\langle T_{1}, \ldots, T_{n}\right\rangle\right) \otimes_{R} K .
$$

We define the category of semiaffinoid $K$-spaces as the opposite of the category of semiaffinoid $K$-algebras, where a morphism of semiaffinoid $K$-algebras is simply a $K$-algebra homomorphism. Semiaffinoid $K$-spaces play the role of "building blocks" for uniformly rigid $K$-spaces, such that we effectively implement Grothendieck's original point of view in the ff type situation. Semiaffinoid $K$ algebras can be studied via the universal properties of the free semiaffinoid $K$ algebras, which we establish in Theorem 2.13.

We define a G-topology on the category of semiaffinoid $K$-spaces equipped with its physical points functor by considering compositions of admissible blowups, completion morphisms and open immersions on flat affine models of ff type; see Definitions 2.22 and 2.31. These formal-geometric constructions define semiaffinoid subdomains, which may be regarded as nested rational subdomains involving strict or nonstrict inequalities in semiaffinoid functions. In contrast to the classical rigid case, we cannot avoid nested constructions; this is essentially due to the fact that admissible blowups defined on open formal subschemes need not extend; see Remark 2.23. Just like in rigid geometry, the disconnected covering of the closed semiaffinoid unit disc sSp $K\langle S\rangle$ by the open semiaffinoid unit disc sSp $K \llbracket S \rrbracket$ and the semiaffinoid unit circle $\operatorname{sip} K\left\langle S, S^{-1}\right\rangle$ is not admissible in the uniformly rigid G-topology; see Example 2.42. In particular, contrary to the rigid-analytic situation, finite coverings of semiaffinoid spaces by semiaffinoid subdomains need not be admissible.

Using methods from formal geometry, we prove a uniformly rigid acyclicity theorem, which in particular implies the following:

Theorem 1.1 (2.41). The presheaf of semiaffinoid functions is a sheaffor the uniformly rigid G-topology.

The resulting functor from the category of semiaffinoid $K$-spaces to the category of locally G-ringed $K$-spaces is fully faithful; hence global uniformly rigid $K$ spaces can be defined; see Definition 2.46. They can be constructed by means of 
standard gluing techniques; this is possible because uniformly rigid spaces satisfy the properties $\left(\mathrm{G}_{0}\right)-\left(\mathrm{G}_{2}\right)$ listed in [Bosch et al. 1984, p. 339]. It follows that the category of uniformly rigid $K$-spaces admits fibered products and that there is a natural generic fiber functor urig from the category of formal $R$-schemes of locally ff type to the category of uniformly rigid $K$-spaces. The final picture can be described as follows:

Theorem 1.2 (Section 2D1). Let $\operatorname{Rig}_{K}, \mathrm{uRig}_{K}$ and $\mathrm{FF}_{R}$ denote the categories of rigid $K$-spaces, of uniformly rigid $K$-spaces and of formal $R$-schemes of locally ff type respectively. Let moreover $\operatorname{Rig}_{K}^{\prime} \subseteq \operatorname{Rig}_{K}$ be the full subcategory of rigid spaces that are quasiparacompact and quasiseparated. There is a diagram of functors

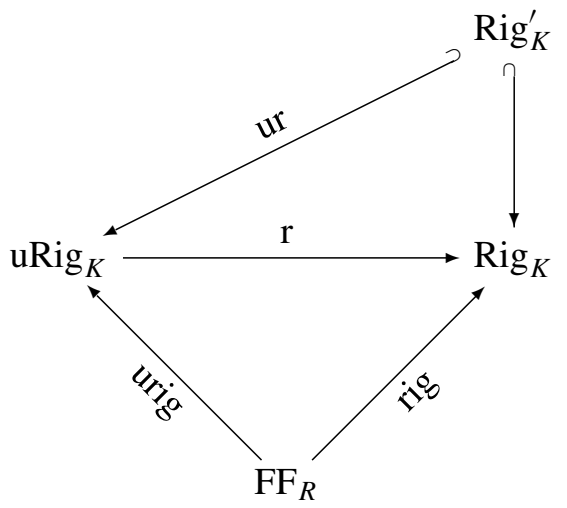

commuting up to isomorphism, where

(i) the functor $\mathrm{r}$ is defined by applying the functor rig locally to models of ff type, where

(ii) the functor ur is defined by applying urig to a global Raynaud-type model of locally tf type

and where the following holds:

(i) The functor ur is a full embedding.

(ii) The functor $\mathrm{r}$ is faithful, yet not fully faithful.

(iii) For each $X \in \mathrm{uRig}_{K}$, there is a comparison morphism $\operatorname{comp}_{X}: X^{r} \rightarrow X$ that is final among all morphisms of locally $G$-ringed $K$-spaces from rigid $K$-spaces to $X$; it is a bijection on physical points, and it induces isomorphisms of stalks.

For $X \in \mathrm{uRig}_{K}$, we say that $X^{\mathrm{r}}$ is the underlying rigid space of $X$. Conversely, for $Y \in \operatorname{Rig}_{K}^{\prime}$ we say that $Y^{\mathrm{ur}}$ is the Raynaud-type uniformly rigid structure on $Y$. Via the comparison morphisms, uniformly rigid spaces and their underlying rigid spaces are locally indistinguishable; we may thus view a uniformly rigid space as a rigid space equipped with an additional global uniform structure which is encoded in terms of a coarser G-topology and a smaller sheaf of analytic functions. Let us 
point out that the open rigid unit disc carries two canonical uniform structures, the one given by a Raynaud model of locally tf type and the one given by the canonical affine model Spf $R \llbracket S \rrbracket$ of ff type. The corresponding uniformly rigid spaces are distinct, since one is not quasicompact while the other one is quasicompact. The fact that $r$ is not fully faithful is seen by the example of an unbounded function $f$ on the rigid open unit disc which we considered above: The rigid-analytic morphism $\varphi$ defined by $f$ does not extend to a morphism of uniformly rigid spaces from $(\text { Spf } R \llbracket S \rrbracket)^{\text {urig }}$ to $\left(\mathbb{P}_{K}^{1, \text { an }}\right)^{\text {ur }}$.

In Section 3, we study coherent modules on uniformly rigid $K$-spaces. We prove the existence of schematic closures of coherent submodules; see Theorem 3.5. Using the resulting models of coherent ideals, we prove the following analog of Kiehl's theorem A in rigid geometry [Kiehl 1967]:

Theorem 1.3 (3.6). Coherent ideals on semiaffinoid spaces are associated to their ideals of global functions.

In particular, closed uniformly rigid subspaces are well-behaved; see Proposition 3.11. Using fibered products and closed uniformly rigid subspaces, we define the notion of separatedness for uniformly rigid $K$-spaces, and we define the graph of a morphism $f: Y \rightarrow X$ of uniformly rigid $K$-spaces whose target is separated; see Section 3A1. Using Theorem 3.5, we show that if $\mathfrak{X}$ and $\mathfrak{Y}$ are flat formal $R$-schemes of locally ff type such that $\mathfrak{X}^{\text {urig }}$ is separated and if $f: \mathfrak{Y}^{\text {urig }} \rightarrow \mathfrak{X}^{\text {urig }}$ is a morphism of uniformly rigid generic fibers, then the schematic closure of the graph of $f$ in $\mathfrak{Y} \times \mathfrak{X}$ exists. As we have noted above, the corresponding statement is false if urig is replaced by Berthelot's generic fiber functor rig.

Semiaffinoid algebras and some associated locally G-ringed $K$-spaces have already been studied in [Lipshitz and Robinson 2000], where the terminology quasiaffinoid is used. The approach in that book includes the situation where $R$ is not discrete and where the machinery of locally noetherian formal geometry is not available. However, no global theory is developed there, and the connection to formal geometry is not discussed. The proof of Theorem 2.13 in the case of a possibly nondiscrete valuation, given in [Lipshitz and Robinson 2000, I.5.2.3], is technically more involved, and it relies upon methods different from ours. The definition of the G-topology in [ibid., III.2.3.2] is less explicit than our definition, so that a deep quantifier elimination theorem [ibid., II, Theorem 4.2] is needed in order to prove an acyclicity theorem. Our approach avoids quantifier elimination.

It is unclear how to reflect uniformly rigid structures on the level of Berkovich's analytic spaces or on the level of Huber's analytic adic spaces; see Section 4. Semiaffinoid $K$-algebras are equipped with unique $K$-Banach algebra structures, so that one may consider their valuation spectra. For instance, the spectrum $M\left(R \llbracket S \rrbracket \otimes_{R} K\right)$ is the closure of the Berkovich open unit disc within the Berkovich closed unit 
disc; it is obtained by adding the Gauss point. However, inclusions of semiaffinoid subdomains need not induce injective maps of valuation spectra, so the formation of the valuation spectrum does not globalize. This corresponds to the fact that in the ff type situation, the functor $\cdot \otimes_{R} K$ does not commute with complete localization. Nonetheless, we suggest that a uniformly rigid $K$-space $X$ should be viewed as a compactification of its underlying rigid space $X^{\mathrm{r}}$. This point of view might be useful in order to obtain a better understanding of the quasicompactifications considered in [Strauch 2008, 3.1] and in [Huber 2007, 3]; it should be further developed within the framework of topos theory. We propose the study of the uniformly rigid topos as a topic for future research.

The author has used uniformly rigid spaces in his doctoral thesis [Kappen 2009], in order to lay the foundations for a theory of formal Néron models of locally ff type. The search for such a theory was strongly motivated by work of C.-L. Chai [2003], who had suggested that Néron models of ff type could be used to study the base change conductor of an abelian variety with potentially multiplicative reduction over a local field. Chai and the author are currently working on further developing the methods of [Chai 2003] within the framework of uniformly rigid spaces.

\section{Uniformly rigid spaces}

Let $R$ be a discrete valuation ring with residue field $k$ and fraction field $K$, and let $\pi \in R$ be a uniformizer.

2A. Formal schemes of formally finite type. A morphism of locally noetherian formal schemes is said to be of locally formally finite (ff) type if the induced morphism of smallest subschemes of definition is of locally finite type. Equivalently, any induced morphism of subschemes of definition is of locally finite type. A morphism of locally noetherian formal schemes is called of ff type if it is of locally ff type and quasicompact. If $A$ is a noetherian adic ring and if $B$ is a noetherian adic topological $A$-algebra, then $\operatorname{Spf} B$ is of ff type over $\operatorname{Spf} A$ if and only if $B$ is a topological quotient of a mixed formal power series ring $A \llbracket S_{1}, \ldots, S_{m} \rrbracket\left\langle T_{1}, \ldots, T_{n}\right\rangle$, where $A \llbracket S_{1}, \ldots, S_{m} \rrbracket$ carries the $\mathfrak{a}+\left(S_{1}, \ldots, S_{m}\right)$-adic topology for any ideal of definition $\mathfrak{a}$ of $A$ [Berkovich 1996, Lemma 1.2]. In this case, we say that the topological $A$-algebra $B$ is of ff type. Morphisms of locally ff type are preserved under composition, base change and formal completion.

We say that an $R$-algebra is of formally finite (ff) type if it admits a ring topology such that it becomes a topological $R$-algebra of ff type in the above sense, where $R$ carries the $\pi$-adic topology. Equivalently, an $R$-algebra is of ff type if it admits a presentation as a quotient of a mixed formal power series ring, as above. If $S$ 
and $T$ are finite systems of variables and if $\varphi: R \llbracket S \rrbracket\langle T\rangle \rightarrow A$ is a surjection, then the $\varphi$-image of $(S, T)$ will be called a formal generating system for $A$.

Lemma 2.1. If $A$ is a topological $R$-algebra of ff type, then the biggest ideal of definition of A coincides with the Jacobson radical of A. Moreover, any $R$ homomorphism of topological R-algebras offf type is continuous.

Proof. Let $\mathfrak{a}$ denote the biggest ideal of definition of $A$; then $\mathfrak{a}$ is contained in every maximal ideal of $A$ since $A$ is $\mathfrak{a}$-adically complete. On the other hand, $A / \mathfrak{a}$ is a Jacobson ring since it is of finite type over the residue field $k$ of $R$; it follows that $\mathfrak{a}$ coincides with the Jacobson radical of $A$, as claimed. In particular, the topology on $A$ is determined by the ring structure of $A$. Let now $A \rightarrow B$ be a homomorphism of $R$-algebras of ff type; by what we have seen so far, it suffices to see that $\varphi$ is continuous for the Jacobson-adic topologies. However, for any maximal ideal $\mathfrak{n} \subseteq B$, the preimage $\mathfrak{m}:=\mathfrak{n} \cap A$ of $\mathfrak{n}$ in $A$ is maximal, since $k \subseteq A / \mathfrak{m} \subseteq B / \mathfrak{n}$, where $B / \mathfrak{n}$ is a finite field extension of $k$ because the quotient $B / \mathrm{jac} B$ is of finite type over $k$.

In particular, the topology on $A$ an be recovered from the ring structure on $A$, and the category of $R$-algebras of ff type is canonically equivalent to the category of topological $R$-algebras of ff type. Lemma 2.1 implies that the category of $R$ algebras of ff type admits amalgamated sums $\hat{\otimes}$.

2B. Semiaffinoid algebras. We define semiaffinoid $K$-algebras as the generic fibers of $R$-algebras of ff type, and we define the category of semiaffinoid $K$-spaces as the dual of the category of semiaffinoid $K$-algebras:

Definition 2.2. Let $A$ be a $K$-algebra.

(i) An $R$-model of $A$ is an $R$-subalgebra $\underline{A} \subseteq A$ such that the natural homomorphism $\underline{A} \otimes_{R} K \rightarrow A$ is an isomorphism.

(ii) The $K$-algebra $A$ is called semiaffinoid if it admits an $R$-model of ff type.

(iii) A homomorphism of semiaffinoid $K$-algebras is a homomorphism of underlying $K$-algebras.

(iv) The category of semiaffinoid $K$-spaces is the dual of the category of semiaffinoid $K$-algebras. If $A$ is a semiaffinoid $K$-algebra, we write $\operatorname{sSp} A$ to denote the corresponding semiaffinoid $K$-space, and if $\varphi: \operatorname{sSp} B \rightarrow \operatorname{sSp} A$ is a morphism of semiaffinoid $K$-spaces, we write $\varphi^{*}$ to denote the corresponding $K$-algebra homomorphism.

By Definition 2.2(i) above, any $R$-model of a $K$-algebra is flat over $R$.

There exists no general analog of the Noether normalization theorem for semiaffinoid $K$-algebras [Lipshitz and Robinson 2000, I.2.3.5]. However, if $A$ is a semiaffinoid $K$-algebra admitting a local $R$-model of ff type, then there exist finitely 
many variables $S_{1}, \ldots, S_{m}$ and a finite $K$-monomorphism

$$
R \llbracket S_{1}, \ldots, S_{m} \rrbracket \otimes_{R} K \hookrightarrow A .
$$

Indeed, if $\underline{A}$ is a local $R$-model of ff type for $A$ with maximal ideal $\mathfrak{m}$ and if $s_{0}, \ldots, s_{m}$ is a system of parameters for $\underline{A}$ such that $s_{0}=\pi$, then there exists a unique continuous $R$-homomorphism $\varphi: R \llbracket S_{1}, \ldots, S_{m} \rrbracket \rightarrow \underline{A}$ sending $S_{i}$ to $s_{i}$, for $1 \leq i \leq m$. If $\mathfrak{r}$ denotes the maximal ideal of $R \llbracket S_{1}, \ldots, S_{m} \rrbracket$, then $\underline{A} / \mathfrak{r} \underline{A}$ is $k$-finite because $\underline{A} / \mathfrak{m} \underline{A}$ is $k$-finite and because $\mathfrak{r}$ is $\mathfrak{m}$-primary. By the formal version of Nakayama's Lemma, cf [Eisenbud 1995, Example 7.2], it follows that $\varphi$ is finite; here we use that $R \llbracket S_{1}, \ldots, S_{m} \rrbracket$ is $\mathfrak{r}$-adically complete and that $\underline{A}$ is $\mathfrak{r}$-adically separated. Since $R \llbracket S_{1}, \ldots, S_{m} \rrbracket$ and $\underline{A}$ have the same dimension, it follows that $\varphi$ is finite, so we obtain the desired finite monomorphism by extending scalars from $R$ to $K$.

2B1. The specialization map. The following statement may be compared with [de Jong 1995, 7.1.9]:

Lemma 2.3. Let $A$ be a semiaffinoid $K$-algebra, and let $\underline{A} \subseteq A$ be an $R$-model of ff type. If $\mathfrak{m}$ is a maximal ideal in $A$, then

$$
\mathrm{sp}_{\underline{A}}(\mathfrak{m}):=\sqrt{(\underline{A} \cap \mathfrak{m})+\pi \underline{A}}
$$

is a maximal ideal in $\underline{A}$, and $A / \mathfrak{m}$ is a finite extension of $K$.

Proof. Let us write $\mathfrak{p}:=\mathfrak{m} \cap \underline{A}$; then $(\underline{A} / \mathfrak{p})_{\pi}=A / \mathfrak{m}$ is a field, and by the ArtinTate theorem [Grothendieck 1964, 0.16.3.3] it follows that $\underline{A} / \mathfrak{p}$ is a semilocal ring of dimension $\leq 1$. Moreover, $\underline{A} / \mathfrak{p}$ is of ff type over $R$ and, hence, $\pi$-adically complete. Since $\underline{A} / \mathfrak{p} \subseteq A / \mathfrak{m}$ is $R$-flat and since $(\underline{A} / \mathfrak{p})_{\pi}$ is local, it thus follows from Hensel's Lemma that $(\underline{A} / \mathfrak{p}) / \pi(\underline{A} / \mathfrak{p})$ is local as well [Bourbaki 1998, III.4.6 Proposition 8]. Since $\mathfrak{p} A=\mathfrak{m}$, the class of $\pi$ in $\underline{A} / \mathfrak{p}$ is nonzero, and so the local noetherian ring $(\underline{A} / \mathfrak{p}) / \pi(\underline{A} / \mathfrak{p})$ is zero-dimensional. Thus its quotient modulo its nilradical is a field, and it follows that the radical of $\mathfrak{p}+\pi \underline{A}$ is maximal in $\underline{A}$, as desired.

To prove that $A / \mathfrak{m}$ is finite over $K$, it suffices to show that $\underline{A} / \mathfrak{p}$ is finite over $R$. Since $R$ is $\pi$-adically complete and since $\underline{A} / \mathfrak{p}$ is $\pi$-adically separated, it thus suffices to show that $\underline{A} /(\mathfrak{p}+\pi \underline{A})$ is finite over $k$ [Eisenbud 1995, Example 7.2]. The ring $\underline{A} /(\mathfrak{p}+\pi \underline{A})$ is noetherian; hence its nilradical is nilpotent, and it thereby suffices to see that the quotient of $\underline{A}$ modulo the maximal ideal $\sqrt{\mathfrak{p}+\pi \underline{A}}$ is $k$ finite. Since $\underline{A}$ is of ff type over $R$, since maximal ideals are open and since field extensions of finite type are finite, the desired statement follows.

Definition 2.4. If $A$ is a semiaffinoid $K$-algebra, we call $|X|:=\operatorname{Max} A$ the set of physical points of its corresponding semiaffinoid $K$-space $X$. We will often write $X$ instead of $|X|$ if no confusion is likely to result. 
Remark 2.5. Lemma 2.3 implies that a morphism $\varphi: \operatorname{sSp} A \rightarrow \operatorname{sSp} B$ induces a map on sets of physical points such that for $R$-models of ff type $\underline{A}$ and $\underline{B}$ with $\varphi^{*}(\underline{B}) \subseteq \underline{A}$, the specialization maps $\operatorname{sp}_{\underline{A}}$ and $\mathrm{sp}_{\underline{B}}$ are compatible with respect to $\varphi$ and the induced morphism $\underline{\varphi}: \operatorname{Spf} \underline{A} \rightarrow \operatorname{Spf} \underline{B}$. This functoriality implies that $\mathrm{sp}_{\underline{A}}$ is surjective onto the set of maximal ideals in $\underline{A}$. Indeed, let $\mathrm{r} \subseteq \underline{A}$ be a maximal ideal, and let $\left.\underline{A}\right|_{\mathrm{r}}$ denote the r-adic completion of $A$; then $\operatorname{Max}\left(\left.\underline{A}\right|_{\mathrm{r}} \otimes_{R} K\right)$ is nonempty, and any element in this set maps to an element in $\operatorname{Max}(A)$ that maps to $\mathrm{r}$ under $\mathrm{sp}_{\underline{A}}$. Let us moreover remark that for $x \in X=\operatorname{sSp} A$ with specialization $\mathfrak{n} \subseteq \underline{A}$, the valuation ring of the residue field of $A$ in $x$ coincides with the integral closure of $\underline{A}_{\mathfrak{n}}$ in that residue field, so that the intersection of $\underline{A}_{\mathfrak{n}}$ with the valuation ideal is precisely $\mathfrak{n} \underline{A}_{\mathfrak{n}}$.

2B2. Power-boundedness and topological quasinilpotency. Let $X$ be a semi-affinoid $K$-space with corresponding semiaffinoid $K$-algebra $A$. By Lemma $2.3, A / \mathfrak{m}$ is $K$-finite for $\mathfrak{m} \subseteq A$ maximal; hence the discrete valuation on $K$ extends uniquely to $A / \mathfrak{m}$, so we can define $|f(x)| \in \mathbb{R}_{\geq 0}$ for any $f \in A, x \in X$.

Definition 2.6. An element $f \in A$ is called power-bounded if $|f(x)| \leq 1$ for all $x \in X$. It is called topologically quasinilpotent if $|f(x)|<1$ for all $x \in X$. We let $\AA \subseteq A$ denote the $R$-subalgebra of power-bounded functions, and we let $\check{A} \subseteq \AA$ denote the ideal of topologically quasinilpotent functions.

For example, $S \in A=R \llbracket S \rrbracket \otimes_{R} K$ is topologically quasinilpotent, while the supremum of the absolute values $|S(x)|$, with $x$ ranging over $X$, is equal to 1 . Thus we see that the classical maximum principle fails for semiaffinoid $K$-algebras. However, the maximum principle holds if we let $x$ vary in the Berkovich spectrum $M(A)$ of $A$, where $A$ is equipped with its unique $K$-Banach algebra topology; see Section 4. Indeed, this follows trivially from the fact that $M(A)$ is compact.

Remark 2.7. If $A$ is a nonreduced semiaffinoid $K$-algebra, then $\AA$ cannot be of ff type over $R$ : If $f \in A$ is a nonzero nilpotent function, then $f \in \AA$ is infinitely $\pi$-divisible in $\AA$, but $R$-algebras of ff type are $\pi$-adically separated.

Remark 2.8. If $\underline{A} \subseteq A$ is an $R$-model of ff type, then $\underline{A} \subseteq \AA$, and $\check{A} \cap \underline{A} \subseteq \underline{A}$ is the biggest ideal of definition. Indeed, by Lemma 2.1 and its proof, the biggest ideal of definition of $\underline{A}$ is given by the Jacobson radical, and hence it suffices to observe that for any $f \in \underline{A}$ and any $x \in \operatorname{sSp} A$ with specialization $\mathfrak{n} \subseteq \underline{A}$, we have $|f(x)| \leq 1$, where $|f(x)|<1$ if and only if $f \in \mathfrak{n}$. This however is clear from the final statement in Remark 2.5.

For the notion of normality for formal $R$-schemes of locally ff type, we refer to the discussion in [Conrad 1999, 1.2], which is based on the fact that $R$-algebras of ff type are excellent. This excellence result is a consequence of [Valabrega 1975, Proposition 7] if $R$ has equal characteristic, and it follows from [Valabrega 
1976, Theorem 9] if $R$ has mixed characteristic. In the following, excellence of $R$-algebras of ff type will be used without further comments.

The following result is fundamental:

Proposition 2.9. Let $A$ be a semiaffinoid $K$-algebra. If $A$ admits a normal $R$ model offf type, then this model coincides with $\AA$.

Proof. Let $\underline{A}$ be a normal $R$-model of ff type for $A$. By [de Jong 1995, 7.1.8], we may view $A$ as a subring of the ring of global functions on $(\operatorname{Spf} \underline{A})^{\mathrm{rig}}$, and by [de Jong 1995, 7.4.1; 1998], $\underline{A}$ coincides with the ring of power-bounded global functions under this identification.

Corollary 2.10. Let $A$ be a semiaffinoid $K$-algebra, and let $\underline{A} \subseteq A$ be an $R$-model offf type; then the inclusion $\underline{A} \subseteq \AA$ is integral. If moreover $A$ is reduced, then this inclusion is finite.

Proof. Let $\underline{\varphi}: \underline{A} \rightarrow \underline{B}$ denote the normalization of $\underline{A}$. Then $\underline{\varphi}$ is finite since $\underline{A}$ is excellent, and hence $\underline{B}$ is of ff type over $R$. Extension of scalars yields an induced homomorphism of semiaffinoid $K$-algebras $\varphi: A \rightarrow B$. Since $\varphi$ factors through an injective $R$-homomorphism $\underline{A} / \operatorname{rad}(\underline{A}) \hookrightarrow \underline{B}$, since $K$ is $R$-flat and since $\operatorname{rad}(A)=\operatorname{rad}(\underline{A}) A$, we see that $\varphi$ factors through an injective $K$-homomorphism $A / \operatorname{rad}(A) \hookrightarrow B$. By Proposition 2.9, $\underline{B}$ coincides with the ring of power-bounded functions in $B$. Let us consider a power-bounded function $f$ in $A$; then $\varphi(f) \in \underline{B}$. Since $\varphi$ is finite, there exists an integral equation $P(T) \in \underline{A}[T]$ for $\varphi(f)$ over $\underline{A}$. By the factorization of $\varphi$ mentioned above, we conclude that $P(f) \in A$ is nilpotent. If $s \in \mathbb{N}$ is an integer such that $P(f)^{s}=0$; then $P(T)^{s} \in \underline{A}[T]$ is an integral equation for $f$ over $\underline{A}$. Finally, if $A$ is reduced, then $\varphi$ is injective, and hence $\AA$ is an $\underline{A}$ submodule of the finite $\underline{A}$-module $\underline{B}$. Since $\underline{A}$ is noetherian, it follows that $\AA$ is a finite $\underline{A}$-module.

We immediately obtain the following:

Corollary 2.11. The ring of power-bounded functions in a reduced semiaffinoid $K$-algebra is a canonical $R$-model of ff type containing any other $R$-model of ff type.

We conclude that any $R$-model of ff type can be enlarged so that it contains any given finite set of power-bounded functions:

Corollary 2.12. Let $\underline{A}$ be an $R$-model of ff type in a semiaffinoid $K$-algebra $A$, and let $M \subseteq A$ be a finite set of power-bounded functions. Then the $\underline{A}$-subalgebra $\underline{A}[M]$ generated by $M$ over $\underline{A}$ is finite over $\underline{A}$ and, hence, an $R$-model of ff type for A.

Proof. The ring extension $\underline{A} \subseteq \underline{A}[M]$ is finite since it is generated by finitely many integral elements. 
2B3. Free semiaffinoid algebras. Using the results of Section 2B2, we can now establish the universal properties of free semiaffinoid $K$-algebras; these are semiaffinoid $K$-algebras of the form $R \llbracket S \rrbracket\langle T\rangle \otimes_{R} K$, for finite systems of variables $S$ and $T$ :

Theorem 2.13. Let $m$ and $n$ be natural numbers. The semiaffinoid $K$-algebra $R \llbracket S_{1}, \ldots, S_{m} \rrbracket\left\langle T_{1}, \ldots, T_{n}\right\rangle \otimes_{R} K$, together with the pair of tuples of functions $\left(\left(S_{1}, \ldots, S_{m}\right),\left(T_{1}, \ldots, T_{n}\right)\right)$, is initial among all semiaffinoid $K$-algebras $A$ equipped with a pair $\left(\left(f_{1}, \ldots, f_{m}\right),\left(g_{1}, \ldots, g_{n}\right)\right)$ satisfying the property that the $g_{j}$ are power-bounded and that the $f_{i}$ are topologically quasinilpotent.

Proof. Let us write $S$ and $T$ to denote the systems of the $S_{i}$ and the $T_{j}$. By Corollary 2.12, $A$ admits an $R$-model of ff type $\underline{A}$ containing the $f_{i}$ and the $g_{j}$. By Remark 2.8, the $f_{i}$ are topologically nilpotent in $\underline{A}$; hence there exists a unique $R$-homomorphism $\varphi: R \llbracket S \rrbracket\langle T\rangle \rightarrow \underline{A}$ sending $S_{i}$ to $f_{i}$ and $T_{j}$ to $g_{j}$ for all $i$ and $j$, and so $\varphi:=\varphi \otimes_{R} \bar{K}$ is a $K$-homomorphism with the desired properties. It remains to show that these properties determine $\varphi$ uniquely. Let $\varphi^{\prime}: R \llbracket S \rrbracket\langle T\rangle \otimes_{R} K \rightarrow A$ be any $K$-homomorphism sending $S_{i}$ to $f_{i}$ and $T_{j}$ to $g_{j}$ for all $i$ and $j$, and let us set $\underline{A}^{\prime}:=\varphi^{\prime}(R \llbracket S \rrbracket\langle T\rangle)$ which is of ff type over $R$. If $\varphi=\varphi^{\prime}$, then $\underline{A}^{\prime} \subseteq \underline{A}$. On the other hand, to show that $\varphi=\varphi^{\prime}$, it suffices to see that, after possibly enlarging $\underline{A}$, we have $\underline{A}^{\prime} \subseteq \underline{A}$, in virtue of the universal property of $R \llbracket S \rrbracket\langle T\rangle$. If $A$ is reduced, Corollary 2.10 says that we may set $\underline{A}$ equal to the ring of power-bounded functions in $A$; in this case the inclusion $\underline{A}^{\prime} \subseteq \underline{A}$ is obvious. In the general case, we let $N$ denote the nilradical of $A$; then, by what we have shown so far,

$$
\underline{A}^{\prime} /\left(\underline{A}^{\prime} \cap N\right) \subseteq \underline{A} /(\underline{A} \cap N)
$$

within $\AA / N$. The ideal $\underline{A}^{\prime} \cap N$ is finitely generated since $\underline{A}^{\prime}$ is noetherian; after enlarging $\underline{A}$ using Corollary 2.12, we may thus assume that $\underline{A}$ contains a generating system $n_{1}, \ldots, n_{r}$ of $\underline{A}^{\prime} \cap N$. The inclusion ( $*$ ) shows that every element $a^{\prime} \in \underline{A}^{\prime}$ is the sum of an element $a \in \underline{A}$ and a linear combination $\sum_{i=1}^{r} a_{i}^{\prime} n_{i}$ with coefficients $a_{i}^{\prime} \in \underline{A}^{\prime}$. Let us write the coefficients $a_{i}^{\prime}$ in the analogous way, and let us iterate the procedure. Using the fact that the $n_{i}$ lie in $\underline{A}$, the only summands possibly not lying in $\underline{A}$ after $s$-fold iteration are multiples of products of the $n_{i}$ involving $s$ factors. Since the $n_{i}$ are nilpotent, these summands are zero for $s$ big enough; hence $\underline{A^{\prime}} \subseteq \underline{A}$, as desired.

With the universal property of the free semiaffinoid $K$-algebras at hand, we can now describe the category of semiaffinoid $K$-algebras in terms of the category of $R$-models of ff type. Let us recall that a formal blowup in the sense of [Temkin $2008,2.1]$ is called admissible if it can be defined by a $\pi$-adically open coherent ideal.

Corollary 2.14. Let $\varphi: A \rightarrow B$ be a homomorphism of semiaffinoid $K$-algebras. 
(i) Let $\underline{A}_{1}, \underline{A}_{2}$ be $R$-models offf type for $A$. If $\underline{A}_{2}$ contains a formal generating system of $\underline{A}_{1}$, then $\underline{A}_{1}$ is contained in $\underline{A}_{2}$.

(ii) An inclusion of $R$-models of ff type for A corresponds to a finite admissible blowup of associated formal spectra.

(iii) Let $\underline{A} \subseteq A, \underline{B} \subseteq B$ be $R$-models of ff type such that there exists a formal generating system of $\underline{A}$ mapping to $\underline{B}$ via $\varphi$. Then $\varphi(\underline{A}) \subseteq \underline{B}$.

(iv) Let $\underline{A}$ be an $R$-model of ff type for $A$. There exists an $R$-model of ff type $\underline{B}$ for $B$ such that $\varphi(\underline{A}) \subseteq \underline{B}$. Moreover, if $\underline{B}^{\prime}$ is any $R$-model of ff type for $B$, we can choose $\underline{B}$ such that $\underline{B}^{\prime} \subseteq \underline{B}$.

Proof. To prove the first statement, let us fix a formal generating system $(f, g)$ of $\underline{A}_{1}$ that is contained in $\underline{A}_{2}$. The components of $f$ are topologically quasinilpotent in $A$; since $\underline{A}_{2}$ is an $R$-model of ff type for $A$, they are topologically nilpotent in $\underline{A}_{2}$. Let $\alpha: R \llbracket S \rrbracket\langle T\rangle \rightarrow \underline{A}_{1}$ and $\beta: R \llbracket S \rrbracket\langle T\rangle \rightarrow \underline{A}_{2}$ be the associated $R$-homomorphisms, where $\alpha$ is surjective because $(f, g)$ formally generates $\underline{A}_{1}$. By Theorem 2.13, $\alpha \otimes_{R} K$ and $\beta \otimes_{R} K$ coincide as homomorphisms from $R \llbracket S \rrbracket\langle T\rangle \otimes_{R} K$ to $A$, so we conclude that $\underline{A}_{1} \subseteq \underline{A}_{2}$ : given $a \in \underline{A}_{1}$, we choose an $\alpha$-preimage $a^{\prime}$ of $a$; then $a=\alpha\left(a^{\prime}\right)=\beta\left(a^{\prime}\right) \in \underline{A}_{2}$.

To prove the second claim, let $\underline{A}_{1} \subseteq \underline{A}_{2}$ be an inclusion of $R$-models of ff type for $A$, and let $M \subseteq \underline{A}_{2}$ be a finite set whose elements are the components of a formal generating system for $\underline{A}_{2}$ over $R$. Then by Corollary 2.12, $\underline{A}_{1}[M] \subseteq \underline{A}_{2}$ is an $R$-model of ff type for $A$ which is finite over $\underline{A}_{1}$. By statement (i), $\underline{A}_{2}=\underline{A}_{1}[M]$ and hence $\underline{A}_{2}$ is finite over $\underline{A}_{1}$. Arguing exactly as in the proof of [Bosch and Lütkebohmert 1993a, 4.5], we see that $\underline{A}_{1} \subseteq \underline{A}_{2}$ corresponds to an admissible formal blowup.

To prove part (iii), let us choose a formal generating system $(f, g)$ of $\underline{A}$ such that the components of $\varphi(f)$ and $\varphi(g)$ are contained in $\underline{B}$. The components of $\varphi(f)$ are topologically nilpotent in $\underline{B}$ since they are topologically quasinilpotent in $B$. Let $\alpha: R \llbracket S \rrbracket\langle T\rangle \rightarrow \underline{A}$ and $\beta: R \llbracket S \rrbracket\langle T\rangle \rightarrow \underline{B}$ be the $R$-homomorphisms defined by $(f, g)$ and $(\varphi(f), \varphi(g))$ respectively; then $\alpha$ is surjective, and Theorem 2.13 shows that $\beta \otimes_{R} K$ coincides with $\varphi \circ\left(\alpha \otimes_{R} K\right)$. As is the proof of statement (i), we conclude that $\varphi(\underline{A}) \subseteq \underline{B}$.

To prove statement (iv), let us choose a formal generating system $(f, g)$ of $\underline{A}$. The components of $\varphi(f)$ are topologically quasinilpotent, and the components of $\varphi(g)$ are power-bounded in $B$. According to Corollary 2.12, there exists an $R$ model $\underline{B}$ of ff type for $B$ containing $\underline{B}^{\prime}$ and the components of $\varphi(f)$ and $\varphi(g)$; by statement (iii), $\varphi(\underline{A}) \subseteq \underline{B}$, as desired.

We can now show that $R$-models of ff type for affinoid $K$-algebras are automatically of tf type: 
Corollary 2.15. Let $A$ be an affinoid $K$-algebra, and let $\underline{A} \subseteq A$ be an $R$-model of ff type. Then $\underline{A}$ is of tf type over $R$.

Proof. Let $\underline{A}^{\prime}$ be an $R$-model of tf type for $A$, and let $\underline{A}^{\prime \prime}$ be an $R$-model of ff type for $A$ containing both $\underline{A}$ and $\underline{A}^{\prime}$; such an $\underline{A}^{\prime \prime}$ exists by Corollary 2.14(iv) applied to the identity on $A$. By Corollary 2.14(ii), $\underline{A}^{\prime \prime}$ is finite over $\underline{A}^{\prime}$ and, hence, an $R$-algebra of tf type. After replacing $\underline{A}^{\prime}$ by $\underline{A}^{\prime \prime}$, we may thus assume that $\underline{A} \subseteq \underline{A^{\prime}}$. Again by Corollary 2.14(ii), this inclusion is finite. We now mimic the proof of the classical Artin-Tate lemma: Let $a_{1}, \ldots, a_{m}$ be a system of topological generators of $\underline{A}^{\prime}$ over $R$, and for each $i$ let $P_{i} \in \underline{A}[T]$ be an integral equation for $a_{i}$ over $\underline{A}$. Let $b_{1}, \ldots, b_{n}$ be the coefficients of the $P_{i}$ in some ordering. Since the $R$-algebra $\underline{A}$ is of ff type, it is $\pi$-adically complete; hence there exists a unique $R$-homomorphism $R\left\langle T_{1}, \ldots, T_{n}\right\rangle \rightarrow \underline{A}$ sending $T_{j}$ to $b_{j}$ for $1 \leq j \leq n$. Let $\underline{B} \subseteq \underline{A}$ denote its image; then $\underline{B}$ is an $R$-algebra of tf type. Since the $a_{i}$ topologically generate $\underline{A}^{\prime}$ over $R$, they also topologically generate $\underline{A}^{\prime}$ over $\underline{B}$. The $a_{i}$ are, by construction, integral over $\underline{B}$; hence $\underline{A}^{\prime}$ is in fact finite over $\underline{B}$. Since $\underline{B}$ is noetherian, the $\underline{B}$-submodule $\underline{A}$ of $\underline{A}^{\prime}$ is finite as well, and it follows that $\underline{A}$ is of tf type as a $\underline{B}$-algebra. We conclude that $\underline{A}$ is of tf type over $R$.

\section{B4. Amalgamated sums.}

Proposition 2.16. The category of semiaffinoid $K$-algebras admits amalgamated sums. More precisely speaking, if $\varphi_{1}: A \rightarrow B_{1}$ and $\varphi_{2}: A \rightarrow B_{2}$ are homomorphisms of semiaffinoid $K$-algebras, then the colimit of the resulting diagram is represented by $\left(\underline{B}_{1} \hat{\otimes}_{A} \underline{B}_{2}\right) \otimes_{R} K$, where $\underline{A}$ and the $\underline{B}_{i}$ are $R$-models offf type for $A$ and the $B_{i}$ respectively such that $\varphi(\underline{A}) \subseteq \underline{B}_{1}, \underline{B}_{2}$.

Proof. By Corollary 2.14(iv), we may choose $R$-models $\underline{A}, \underline{B}_{1}$ and $\underline{B}_{2}$ as in the statement of the proposition. Let $C$ be a semiaffinoid $K$-algebra, and for $i=1,2$ let $\tau_{i}: B_{i} \rightarrow C$ be a $K$-homomorphism such that $\tau_{1} \circ \varphi_{1}=\tau_{2} \circ \varphi_{2}$. By Corollary 2.14(iv), there exists an $R$-model $\underline{C}$ of ff type for $C$ such that $\tau_{i}\left(\underline{B}_{i}\right) \subseteq \underline{C}$ for $i=1,2$; we let $\underline{\tau}_{i}: \underline{B}_{i} \rightarrow \underline{C}$ denote the induced $R$-homomorphism. Then $\underline{\tau}_{1} \circ \underline{\varphi}_{1}=\underline{\tau}_{2} \circ \underline{\varphi}_{2}$, since the same holds after inverting $\pi$ and since $\pi$ is not a zero divisor in $\underline{A}$. By the universal property of the complete tensor product in the category of $R$-algebras of ff type, there exists a unique $R$-homomorphism $\underline{\tau}: \underline{B}_{1} \hat{\otimes}_{\underline{A}} \underline{B}_{2} \rightarrow \underline{C}$ such that $\underline{\tau}_{i}=\underline{\tau} \circ \underline{\sigma}_{i}$ for $i=1,2$, where $\underline{\sigma}_{i}: \underline{B}_{i} \rightarrow \underline{B}_{1} \hat{\otimes}_{\underline{A}} \underline{B}_{2}$ is the $i$ th coprojection. Setting $\tau:=\underline{\tau} \otimes_{R} K$ and $\sigma_{i}:=\underline{\sigma}_{i} \otimes_{R} K$, we obtain $\tau_{i}=\tau \circ \sigma_{i}$ for $i=1$, 2. We must show that $\tau$ is uniquely determined by this property. Let

$$
\tau^{\prime}:\left(\underline{B}_{1} \hat{\otimes}_{A} \underline{B}_{2}\right) \otimes_{R} K \rightarrow C
$$

be any $K$-homomorphism satisfying $\tau_{i}=\tau^{\prime} \circ \sigma_{i}$ for $i=1,2$. By Corollary 2.14(iv), there exists an $R$-model $\underline{C}^{\prime}$ of ff type for $C$ containing $\underline{C}$ such that $\tau^{\prime}$ restricts to an $R$-morphism $\underline{\tau}^{\prime}: \underline{B}_{1} \hat{\otimes}_{\underline{A}} \underline{B}_{2} \rightarrow \underline{C}^{\prime}$; then $\tau^{\prime}=\underline{\tau}^{\prime} \otimes_{R} K$. It suffices to show that 
$\underline{\tau}^{\prime}$ coincides with $\underline{\tau}$ composed with the inclusion $\underline{\imath}: \underline{C} \subseteq \underline{C}^{\prime}$. For $i=1,2$, the compositions $\underline{\tau}^{\prime} \circ \underline{\sigma}_{i}$ and $\underline{\imath} \circ \underline{\tau} \circ \underline{\sigma}_{i}$ coincide after inverting $\pi$, hence they coincide because $\pi$ is not a zero divisor in $\underline{B}_{i}$, for $i=1,2$. The universal property of $\left(\underline{B}_{1} \hat{\otimes}_{\underline{A}} \underline{B}_{2}, \underline{\sigma}_{1}, \underline{\sigma}_{2}\right)$ implies that $\underline{\tau}^{\prime}=\underline{\iota} \circ \underline{\tau}$, as desired.

Passing to the opposite category, we see that the category of semiaffinoid $K$ spaces has fibered products.

2B5. The Nullstellensatz.

Proposition 2.17. Semiaffinoid $K$-algebras are Jacobson rings.

Proof. Any quotient of a semiaffinoid $K$-algebra is again semiaffinoid; hence it suffices to show that if $A$ is a semiaffinoid $K$-algebra and if $f \in A$ is a semiaffinoid function such that $f(x)=0$ for all $x \in \operatorname{sSp} A$, then $f$ is nilpotent. We may divide $A$ by its nilradical and thereby assume that $A$ is reduced. Let $\underline{A}$ be an $R$-model of ff type for $A$, and let $X=(\operatorname{Spf} \underline{A})^{\text {rig }}$ denote the rigid-analytic generic fiber of Spf $\underline{A}$. Since $A$ is excellent, being a localization of the excellent ring $\underline{A}$, and since rigid $K$-spaces are excellent [Conrad 1999, 1.1], it follows from [de Jong 1995 , Lemma 7.1.9] that the space $X$ is reduced and that we may view $A$ as a subring of $\Gamma\left(X, O_{X}\right)$ such that the value of $f$ in a point $x \in X$ agrees with the value of $f$ in the corresponding maximal ideal of $A$. Since $f(x)=0$ for all $x \in X$, we see that $f=0$ as a function on $X$ and, hence, in $A$.

\section{C. Semiaffinoid spaces.}

2C1. The rigid space associated to a semiaffinoid $K$-space. Let $X=\operatorname{sSp} A$ be a semiaffinoid $K$-space. An affine flat formal model of ff type for $X$ is an affine flat formal $R$-scheme of ff type $\mathfrak{X}$ together with an identification of $\Gamma\left(\mathfrak{X}, \mathcal{O}_{\mathfrak{X}}\right)$ with an $R$-model of ff type for $A$. By Definition 2.2, every semiaffinoid $K$-space admits an affine flat model of $\mathrm{ff}$ type. There is an obvious generic fiber functor urig from the category of affine flat formal $R$-schemes of ff type to the category of semiaffinoid $K$-spaces, given by

$$
(\operatorname{Spf} \underline{A})^{\text {urig }}:=\operatorname{sSp}\left(\underline{A} \otimes_{R} K\right) .
$$

Let $\mathfrak{X}$ be a flat affine $R$-model of ff type for $X$. Berthelot's construction yields a rigid $K$-space $X^{\mathrm{r}}:=\mathfrak{X}^{\text {rig }}$ together with a $K$-homomorphism

$$
\varphi: A \rightarrow \Gamma\left(X^{\mathrm{r}}, \mathrm{O}_{X^{\mathrm{r}}}\right)
$$

see [de Jong 1995, 7.1.8]. By our discussion in Section 2B1 and by [ibid., 7.1.9] the homomorphism $\varphi$ induces a bijection $\left|X^{\mathrm{r}}\right| \rightarrow|X|$ and local homomorphisms $A_{\mathfrak{m}} \rightarrow \mathrm{O}_{X^{\mathrm{r}}, x}$ which are isomorphisms on maximal-adic completions, where $x$ is a point of $X^{\mathrm{r}}$ and where $\mathfrak{m} \in \operatorname{Max} A$ is the image of $x$ under the above bijection. We say that $X^{\mathrm{r}}$ is the rigid space associated to $X$ via Berthelot's construction. 
It is independent of the choice of $\mathfrak{X}$, the pair $\left(X^{\mathrm{r}}, \varphi\right)$ being characterized by the following universal property:

Proposition 2.18. Let $Y$ be a rigid $K$-space, and let $\psi: A \rightarrow \Gamma\left(Y, O_{Y}\right)$ be a $K$-algebra homomorphism. There exists a unique morphism of rigid $K$-spaces $\sigma: Y \rightarrow X^{\mathrm{r}}$ such that $\psi=\Gamma\left(\sigma^{\sharp}\right) \circ \varphi$.

Proof. Uniqueness of $\sigma$ follows from the above-mentioned fact that $\varphi$ induces a bijection of points and isomorphisms of completed stalks; we may thus assume that $Y$ is affinoid, $Y=\operatorname{Sp} B$. Let $\underline{A} \subseteq A$ be the $R$-model of ff type corresponding to $\mathfrak{X}$. By Corollary 2.14(iv) and Corollary $2.15, \psi$ restricts to an $R$-homomorphism $\psi: \underline{A} \rightarrow \underline{B}$, where $\underline{B}$ is a suitable $R$-model of tf type for $B$; now $\sigma:=(\operatorname{Spf} \psi)^{\text {rig }}$ has the required properties.

If $\underline{\tau}: \mathfrak{Y} \rightarrow \mathfrak{X}$ is a morphism of affine flat formal $R$-schemes of ff type and if $\underline{\tau}^{\text {urig }}$ denotes the induced morphism of associated semiaffinoid $K$-spaces, we easily see that the unique morphism $\left(\underline{\tau}^{\text {urig }}\right)^{r}$ provided by Proposition 2.18 is given by $\underline{\tau}^{\text {rig }}$.

2C2. Semiaffinoid subdomains. Closed subspaces of semiaffinoid $K$-spaces are easily defined in the usual way:

Definition 2.19. A morphism of semiaffinoid $K$-spaces is called a closed immersion if it corresponds to a surjective homomorphism of semiaffinoid $K$-algebras. A closed semiaffinoid subspace of a semiaffinoid $K$-space is an equivalence class of closed immersions, where two closed immersions of uniformly rigid $K$-spaces $i_{1}: Y_{1} \rightarrow X$ and $i_{2}: Y_{2} \rightarrow X$ are called equivalent if there exists an isomorphism $\varphi: Y_{1} \stackrel{\sim}{\rightarrow} Y_{2}$ such that $i_{1}=i_{2} \circ \varphi$.

If $A$ is a semiaffinoid $K$-algebra and if $I \subseteq A$ is an ideal, then the natural closed immersion $\mathrm{sSp} A / I \rightarrow \mathrm{sSp} A$ is clearly injective onto the set of maximal ideals containing $I$. Moreover, if $A \rightarrow C$ is a homomorphism of semiaffinoid $K$-algebras, then $A / I \hat{\otimes}_{A} C=C / I C$, because this quotient already represents the amalgamated sum of $C$ and $A / I$ over $A$ in the category of all $K$-algebras. In particular, closed immersions of semiaffinoid $K$-spaces are stable under the formation of fibered products.

To define a reasonable structure of G-topological $K$-space on the set of physical points of a semiaffinoid $K$-space $X$, it is natural to consider subsets $U$ of $X$ that canonically inherit a structure of semiaffinoid $K$-space:

Definition 2.20. A subset $U$ in a semiaffinoid $K$-space $X$ is called representable if there exists a morphism of semiaffinoid $K$-spaces to $X$ whose image lies in $U$ and which is final with this property. Such a morphism is said to represent all semiaffinoid morphisms to $X$ with image in $U$. 
Remark 2.21. Here we differ from the terminology used in the author's $\mathrm{PhD}$ thesis; there the representable subsets are called semiaffinoid presubdomains [Kappen 2009, Section 1.3.3].

Clearly $X$ and $\varnothing$ are representable subsets of $X$. Copying the proof of [Bosch et al. 1984, 7.2.2/1], we see that a morphism representing a subset $U \subseteq X$ is injective with image $U$ and that it induces isomorphisms of infinitesimal neighborhoods of points. Using the existence of fibered products in the category of semiaffinoid $K$-spaces, we see that representable subsets are preserved under pullback with respect to morphisms of semiaffinoid spaces. The universal property of representable subsets yields a presheaf $O_{X}$ in semiaffinoid $K$-algebras on the category of representable subsets in $X$.

In the category of affinoid $K$-spaces, the representable subsets are called affinoid subdomains [Bosch et al. 1984, 7.2.2/2], and they play a predominant role in the foundations of rigid geometry. In the uniformly rigid setting, we are unable to handle general representable subsets; for instance, we do not know whether representable subsets induce admissible open subsets via the functor $r$ which is induced by Berthelot's construction. We will thus only consider representable subsets of a specific kind, which we call semiaffinoid subdomains:

Definition 2.22. A subset $U$ of a semiaffinoid $K$-space $X$ is called a semiaffinoid subdomain if there is an affine flat $R$-model of ff type $\mathfrak{X}$ for $X$ and a finite composition of open immersions, completion morphisms and admissible blowups $\varphi: \mathfrak{U} \rightarrow \mathfrak{X}$ such that $\mathfrak{U}$ is affine and such that $U$ is equal to the image of $\varphi^{\text {urig }}$. We say that $\varphi$ represents $U$ as a semiaffinoid subdomain in $X$. We say that $U$ is an elementary semiaffinoid subdomain in $X$ if $\varphi$ can be chosen as an open immersion into an admissible blowup, and we say that $U$ is a retrocompact semiaffinoid subdomain in $X$ if $\varphi$ can be chosen as a composition of open immersions and admissible blowups; such a $\varphi$ is said to represent $U$ as an elementary or as a retrocompact semiaffinoid subdomain in $X$ respectively.

In Corollary 2.25, we will see that semiaffinoid subdomains are actually representable in the sense of Definition 2.20.

Open immersions of formal $R$-schemes of ff type induce retrocompact open immersions of rigid generic fibers [de Jong 1995, 7.2.2 and 7.2.4(d)]. Moreover, completion morphisms induce (possibly nonretrocompact) open immersions of rigid generic fibers [de Jong 1995, 7.2.5], and admissible blowups induce isomorphisms of rigid generic fibers; see [Nicaise 2009, 2.19]. Hence a semiaffinoid subdomain $U \subseteq X$ is admissibly open in $X^{\mathrm{r}}$. In particular, the $K$-homomorphism $\varphi^{\text {urig,* }}$ corresponding to $\varphi^{\text {urig }}$ is flat, since flatness is seen on the level of completions of stalks. Semiaffinoid subdomains may be regarded as nested rational subdomains defined in terms of strict or nonstrict inequalities involving semiaffinoid functions. 
For example, the blowup of $\mathfrak{X}=\operatorname{Spf} R \llbracket S \rrbracket$ in the ideal $(\pi, S)$ is covered by the affine open formal subschemes $\mathfrak{X}_{1}=\operatorname{Spf}(R \llbracket S \rrbracket\langle V\rangle /(\pi V-S)) \cong \operatorname{Spf} R\langle V\rangle$ and $\mathfrak{X}_{2}=\operatorname{Spf}(R \llbracket S \rrbracket\langle W\rangle /(S W-\pi))$; the completion of $\mathfrak{X}_{1}$ along the ideal $(\pi, V)$ represents the open disc with radius $|\pi|$ within the open unit disc, while the completion of $\mathfrak{X}_{2}$ along $(\pi, W)$ defines the open annulus $|\pi|<|S|<1$.

Remark 2.23. It is necessary to consider iterations as in Definition 2.22 because if $\mathfrak{U}$ is an open subset of a flat formal $R$-scheme of ff type $\mathfrak{X}$, then an admissible blowup of $\mathfrak{U}$ needs not extend to an admissible blowup of $\mathfrak{X}$; see [Kappen 2009, Example 1.1.3.12].

In order to understand semiaffinoid subdomains, it will be useful to interpret strict transforms with respect to admissible blowups as pullbacks:

Lemma 2.24. Let $\mathfrak{Y} \rightarrow \mathfrak{X}$ be a morphism of flat formal $R$-schemes of locally ff type, let $\mathfrak{X}^{\prime} \rightarrow \mathfrak{X}$ be an admissible blowup, and let $\mathfrak{Y}^{\prime} \rightarrow \mathfrak{Y}$ denote the induced admissible blowup of $\mathfrak{Y}$, that is, the strict transform of $\mathfrak{Y}$. The resulting square

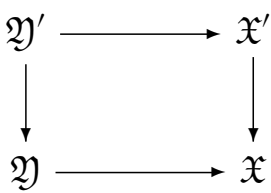

is cartesian in the category of flat formal $R$-schemes of locally ff type.

Proof. The universal property of the fibered product in the category of flat formal $R$-schemes of locally ff type is readily verified using the universal property of admissible blowups, the fact that the functor rig maps admissible blowups to isomorphisms and the fact that rig is faithful on the category of flat formal $R$-schemes of locally ff type.

In the following, we write $x^{\prime}$ to denote the fibered product in the category of flat formal $R$-schemes of locally ff type. It is obtained from the usual fibered product by dividing out the coherent ideal of $\pi$-torsion; in particular, fibered products of affine flat formal $R$-schemes of ff type in the category of flat formal $R$-schemes of locally ff type are again affine.

As we have just observed, admissible blowups of flat formal $R$-schemes are preserved under pullback in the category of flat formal $R$-schemes of locally $\mathrm{ff}$ type. The same is true for open immersions and completion morphisms, since they are flat and since they are preserved under pullback in the category of all formal $R$-schemes of locally ff type.

Corollary 2.25. Let $X$ be a semiaffinoid $K$-space, let $U \subseteq X$ be a semiaffinoid subdomain, and let $Y \rightarrow X$ be a morphism of semiaffinoid $K$-spaces.

(i) The preimage of $U$ in $Y$ is a semiaffinoid subdomain in $Y$. 
(ii) If $\mathfrak{U} \rightarrow \mathfrak{X}$ represents $U$ as a semiaffinoid subdomain in $X$ and if $\mathfrak{Y} \rightarrow \mathfrak{X}$ is a model of $Y \rightarrow X$, then the projection $\mathfrak{U} \times_{\mathfrak{X}}^{\prime} \mathfrak{Y} \rightarrow \mathfrak{Y}$ represents the preimage of $U$ as a semiaffinoid subdomain in $Y$.

(iii) If $\varphi$ represents $U$ as a semiaffinoid subdomain in $X$, then $\varphi^{\text {urig }}$ represents all semiaffinoid morphisms to $X$ with image in $U$. In particular, semiaffinoid subdomains are representable in the sense of Definition 2.20.

The analogous statements hold if we consider retrocompact or elementary semiaffnoid subdomains and their retrocompact or elementary representations.

Proof. Statement (ii) implies statement (i) in view of Corollary 2.14(iv). To show (ii), let us consider a factorization

$$
\mathfrak{U} \stackrel{\varphi_{n}}{\longrightarrow} \mathfrak{U}_{n} \stackrel{\varphi_{n-1}}{\longrightarrow} \cdots \stackrel{\varphi_{1}}{\longrightarrow} \mathfrak{U}_{1} \stackrel{\varphi_{0}}{\longrightarrow} \mathfrak{X}
$$

of $\mathfrak{U} \rightarrow \mathfrak{X}$, where the $\varphi_{i}$ are admissible blowups, open immersions or completion morphisms. By the remarks preceding this Corollary, we see that the projection $\mathfrak{U} \times_{\mathfrak{X}}^{\prime} \mathfrak{Y} \rightarrow \mathfrak{Y}$ defines a semiaffinoid subdomain in $Y$. Passing to associated rigid spaces, we see that this semiaffinoid subdomain coincides with the preimage of $U$ in $Y$. To prove (iii), let us write $\varphi$ to denote $\mathfrak{U} \rightarrow \mathfrak{X}$, and let us assume that the image of $Y \rightarrow X$ lies in $U$; we must show that $Y \rightarrow X$ factors uniquely through $\varphi^{\text {urig }}$. Since $\varphi^{\text {urig }}$ induces an injection of physical points and isomorphisms of completed stalks, uniqueness follows from Krull's Intersection theorem. Let us show that the desired factorization exists. Again, Corollary 2.14(iv) shows that $Y \rightarrow X$ admits a model $\mathfrak{Y} \rightarrow \mathfrak{X}$ with target $\mathfrak{X}$. Let us consider the pullback

$$
\mathfrak{Y}_{n+1} \stackrel{\psi_{n}}{\longrightarrow} \mathfrak{Y}_{n} \stackrel{\psi_{n-1}}{\longrightarrow} \cdots \stackrel{\psi_{1}}{\longrightarrow} \mathfrak{Y}_{1} \stackrel{\varphi_{0}}{\longrightarrow} \mathfrak{Y}
$$

of $(\dagger)$ under $\mathfrak{Y} \rightarrow \mathfrak{X}$ in the category of flat formal $R$-schemes of locally ff type; then $\mathfrak{Y}_{n+1}$ is affine, and all $\psi_{i}$ that are open immersions or completion morphisms are isomorphisms: Indeed, $Y \rightarrow X$ factors through $U$, specialization maps are surjective onto the sets of closed points of flat formal $R$-schemes of locally ff type, and the closed points lie very dense in formal $R$-schemes of this type. Hence, the composition $\mathfrak{Y}_{n+1} \rightarrow \mathfrak{Y}$ is a composition of admissible blowups; by [Temkin 2008, 2.1.6], it is an admissible blowup. Since $\mathfrak{Y}_{n+1}$ is affine, [Grothendieck 1961b, 3.4.2] shows that $\mathfrak{Y}_{n+1} \rightarrow \mathfrak{Y}$ is a finite admissible blowup. After applying urig, we thus obtain the desired factorization of $Y \rightarrow X$.

By Corollary 2.25(iii), every semiaffinoid subdomain may be viewed as a semiaffinoid $K$-space in a natural way.

Question 2.26. One may ask whether every representable subset of a semiaffinoid $K$-space is in fact a semiaffinoid subdomain. Unfortunately, we do not know the answer. 
Corollary 2.27. Let $X$ be a semiaffinoid $K$-space, let $U \subseteq X$ be a semiaffinoid subdomain, and let $\mathfrak{X}$ be a flat affine $R$-model offf type for $X$. Then there exists a representation of $U$ as a semiaffinoid subdomain in $X$ with target $\mathfrak{X}$.

Proof. Let $\mathfrak{U}^{\prime} \rightarrow \mathfrak{X}^{\prime}$ be a representation of $U$ as a semiaffinoid subdomain in $X$, let us write $X=\mathrm{sSp} A$, and let $\underline{A}, \underline{A}^{\prime} \subseteq A$ be the $R$-models of ff type of $A$ corresponding to $\mathfrak{X}$ and $\mathfrak{X}^{\prime}$ respectively. By Corollary 2.14(iv) applied to the identity on $A$, there exists an $R$-model of ff type $\underline{A}^{\prime \prime}$ of $A$ containing both $\underline{A}$ and $\underline{A}^{\prime}$. By Corollary 2.14(ii), the inclusions $\underline{A} \subseteq \underline{A}^{\prime \prime}$ and $\underline{A}^{\prime} \subseteq \underline{A}^{\prime \prime}$ correspond to finite admissible blowups $\mathfrak{X}^{\prime \prime} \rightarrow \mathfrak{X}$ and $\mathfrak{X}^{\prime \prime} \rightarrow \mathfrak{X}^{\prime}$. By Corollary 2.25(ii), the strict transform $\mathfrak{U}^{\prime \prime} \rightarrow \mathfrak{X}^{\prime \prime}$ of $\mathfrak{U}^{\prime} \rightarrow \mathfrak{X}^{\prime}$ under $\mathfrak{X}^{\prime \prime} \rightarrow \mathfrak{X}^{\prime}$ represents $U$ as a semiaffinoid subdomain in $X$. Composing this representation with the admissible blowup $\mathfrak{X}^{\prime \prime} \rightarrow \mathfrak{X}$, we obtain a representation $\mathfrak{U}^{\prime \prime} \rightarrow \mathfrak{X}$ of $U$ as a semiaffinoid subdomain in $X$ with target $\mathfrak{X}$, as desired.

Remark 2.28. One can easily show that if $U \subseteq X$ is a semiaffinoid subdomain and if $\mathfrak{Y} \rightarrow \mathfrak{X}$ is a model of the inclusion of $U$ into $X$, then there exists a finite admissible blowup $\mathfrak{Y}^{\prime}$ of $\mathfrak{Y}$ such that the composition $\mathfrak{Y}^{\prime} \rightarrow \mathfrak{X}$ represents $U$ as a semiaffinoid subdomain in $X$; this fact will not be needed in the following.

Corollary 2.29. Let $X$ be a semiaffinoid $K$-space.

(i) Let $U \subseteq X$ be a semiaffinoid subdomain, and let $V$ be a subset of $U$. Then $V$ is a semiaffinoid subdomain in $U$ if and only if it is a semiaffinoid subdomain in $X$.

(ii) The set of semiaffinoid subdomain in $X$ is stable under the formation of finite intersections.

Proof. If $V$ is semiaffinoid in $X$, then $V=V \cap U$ is semiaffinoid in $U$ by Corollary 2.25(i). Conversely, assume that $V$ is semiaffinoid in $U$, and let $\mathfrak{U} \rightarrow \mathfrak{X}$ be a representation of $U$ as a semiaffinoid subdomain in $X$. By Corollary 2.27, there exists a representation $\mathfrak{V} \rightarrow \mathfrak{U}$ of $V$ as a semiaffinoid subdomain in $U$; the composition $\mathfrak{V} \rightarrow \mathfrak{U} \rightarrow \mathfrak{X}$ represents $V$ as a semiaffinoid subdomain in $X$. This settles the first statement. To show (ii), let us consider two semiaffinoid subdomains $U$ and $V$ in $X$. By Corollary 2.25(i), $U \cap V$ is a semiaffinoid subdomain in $U$; by part (i), $U \cap V$ is thus a semiaffinoid subdomain in $X$.

These results obviously remain true if we only consider retrocompact semiaffinoid subdomains instead of general semiaffinoid subdomains. Similarly, elementary semiaffinoid subdomains are preserved under pullback with respect to morphisms of semiaffinoid spaces. However, if $U$ is an elementary semiaffinoid subdomain in a semiaffinoid $K$-space $X$ and if $V$ is an elementary semiaffinoid 
subdomain in $U$, then $V$ needs not be elementary in $X$. Likewise, if $U$ is a semiaffinoid subdomain in $X$ and if $V$ is a retrocompact semiaffinoid subdomain in $U$, then $V$ needs not be retrocompact in $X$.

We conclude this section by identifying retrocompact semiaffinoid subdomains in affinoid $K$-spaces:

Lemma 2.30. Let $A$ be an affinoid $K$-algebra; then a retrocompact semiaffinoid subdomain $U$ in $\mathrm{sSp} A$ is an affinoid subdomain in $\mathrm{Sp} A$.

Proof. Let $\varphi: \mathfrak{Y} \rightarrow \mathfrak{X}$ be a morphism defining $U$ as a retrocompact semiaffinoid subdomain in $X$. By Corollary $2.15, \mathfrak{X}$ is of tf type over $R$. Since $\varphi$ is adic, $\mathfrak{Y}$ is of tf type over $R$ as well, such that $\varphi^{\text {rig }}$ is a morphism of affinoid $K$-spaces. By Corollary 2.25(iii), $\varphi$ represents all semiaffinoid maps with image in $U$; in particular it represents all affinoid maps with image in $U$. Hence, $U$ is an affinoid subdomain in $\operatorname{Sp} A$.

Conversely, it is clear that for any affinoid $K$-algebra $A$, the rational subdomains in $\operatorname{Sp} A$ define semiaffinoid subdomains in $\operatorname{sSp} A$. Let $U \subseteq \operatorname{Sp} A$ be a general affinoid subdomain in $\mathrm{Sp} A$. By the theorem of Gerritzen and Grauert [Bosch et al. $1984,7.3 .5 / 1$ ], $U$ is a finite union of rational subdomains. Let $\mathfrak{X}$ be any affine flat formal $R$-model of tf type for Sp A. By [Bosch and Lütkebohmert 1993a, Lemma 4.4], there exist an admissible formal blowup $\mathfrak{X}^{\prime} \rightarrow \mathfrak{X}$ of $\mathfrak{X}$ and an open formal subscheme $\mathfrak{U} \subseteq \mathfrak{X}^{\prime}$ such that $U=\mathfrak{U}^{\text {rig. }}$. However, we do not know whether $\mathfrak{U}$ is affine, so we do not know whether a general affinoid subdomain $U$ in $\operatorname{Sp} A$ is a semiaffinoid subdomain or even a representable subset in $\mathrm{sSp} A$. Nonetheless, we will see that affinoid subdomains in $\mathrm{Sp} A$ are admissible open in the uniformly rigid G-topology on $\mathrm{sSp} A$; see Proposition 2.34.

2C3. G-topologies on semiaffinoid spaces. We first define an auxiliary G-topo$\operatorname{logy} \mathscr{T}_{\text {aux }}$ on the category of semiaffinoid $K$-spaces equipped with the physical points functor; see [Bosch et al. 1984, 9.1.2]. The $\mathscr{T}_{\text {aux }}$-admissible subsets of a semiaffinoid $K$-space are the semiaffinoid subdomains of that space. If $I$ is a rooted tree and if $i \in I$ is a vertex, we let $\operatorname{ch}(i)$ denote the set of children of $i$.

Definition 2.31. Let $X$ be a semiaffinoid $K$-space, and let $\left(X_{i}\right)_{i \in I}$ be a finite family of semiaffinoid subdomains in $X$.

(i) We say that $\left(X_{i}\right)_{i \in I}$ is an elementary covering of $X$ if there exist an affine flat $R$-model of ff type $\mathfrak{X}$ for $X$, an admissible blowup $\mathfrak{X}^{\prime} \rightarrow \mathfrak{X}$ and an affine open covering $\left(\mathfrak{X}_{i}\right)_{i \in I}$ of $\mathfrak{X}^{\prime}$ such that for each $i \in I, \mathfrak{X}_{i} \subseteq \mathfrak{X}^{\prime} \rightarrow \mathfrak{X}$ represents $X_{i}$ as a semiaffinoid subdomain in $X$.

(ii) We say that $\left(X_{i}\right)_{i \in I}$ is a treelike covering of $X$ if there exists a rooted tree structure on $I$ such that $X_{r}=X$, where $r$ is the root of $I$, and such that 
$\left(X_{j}\right)_{j \in \operatorname{ch}(i)}$ is an elementary covering of $X_{i}$ for all $i \in I$ which are not leaves. A rooted tree structure on $I$ with these properties is called suitable for $\left(X_{i}\right)_{i \in I}$.

(iii) We say that $\left(X_{i}\right)_{i \in I}$ is a leaflike covering if it extends to a treelike covering $\left(X_{i}\right)_{i \in J}, J \supseteq I$, where $J$ admits a suitable rooted tree structure such that $I$ is identified with the set of leaves of $J$.

(iv) We say that $\left(X_{i}\right)_{i \in I}$ is $\mathscr{T}_{\text {aux }}$-admissible if it admits a leaflike refinement.

If $\left(X_{i}\right)_{i \in I}$ is an elementary, treelike or leaflike covering of $X$, then by definition all $X_{i}$ are retrocompact in $X$. For trivial reasons, condition (iv) in Definition 2.31 can be checked after refinement.

Arguing as in the proof of Corollary 2.27, we see that an elementary covering can be represented with respect to any flat affine $R$-model of ff type $\mathfrak{X}$ of $X$. It follows that any treelike covering $\left(X_{i}\right)_{i \in I}$ together with a suitable rooted tree structure on $I$ admits a model with respect to $\mathfrak{X}$; that is, we have

(i) for each $i \in I$, an affine flat $R$-model of ff type $\mathfrak{X}_{i}$ for $X_{i}$ such that $\mathfrak{X}_{r}=\mathfrak{X}$, where $r$ denotes the root of $I$,

(ii) for each inner $i \in I$, an admissible blowup $\mathfrak{X}_{i}^{\prime} \rightarrow \mathfrak{X}_{i}$ and

(iii) for each inner $i \in I$ and for each child $j$ of $i$, an open immersion $\mathfrak{X}_{j} \hookrightarrow \mathfrak{X}_{i}^{\prime}$ such that $\mathfrak{X}_{j} \subseteq \mathfrak{X}_{i}^{\prime} \rightarrow \mathfrak{X}_{i}$ represents $X_{j}$ as a semiaffinoid subdomain in $X_{i}$.

Arguing as in the proof of Corollary 2.25, we see that elementary, treelike and leaflike coverings, suitable rooted tree structures and models in the above sense are preserved under pullback with respect to morphisms $Y \rightarrow X$ of semiaffinoid $K$-spaces and their models $\mathfrak{Y} \rightarrow \mathfrak{X}$, where $\mathfrak{Y}$ and $\mathfrak{X}$ are flat affine models of ff type for $Y$ and $X$ respectively.

Lemma 2.32. Let $X$ be a semiaffinoid $K$-space, let $\left(U_{i}\right)_{i \in I}$ be a covering of $X$ by semiaffinoid subdomains, and for each $i \in I$, let $\left(V_{i j}\right)_{j \in J_{i}}$ be a covering of $U_{i}$. If all of these coverings are leaflike or $\mathscr{T}_{\text {aux }}$-admissible, then the same holds for the covering $\left(V_{i j}\right)_{i \in I, j \in J_{i}}$ of $X$.

Proof. Let us first consider the case where the given coverings are leaflike. Let us choose a treelike covering $\left(U_{i}\right)_{i \in I^{\prime}}$ of $U$ extending $\left(U_{i}\right)_{i \in I}$ together with a suitable rooted tree structure on $I^{\prime}$ such that $I \subseteq I^{\prime}$ is the set of leaves. Similarly, for each $i \in I$ we choose a treelike covering $\left(V_{i j}\right)_{j \in J_{i}^{\prime}}$ extending $\left(V_{i j}\right)_{j \in J_{i}}$ together with a suitable rooted tree structure on $J_{i}^{\prime}$ such that $J_{i} \subseteq J_{i}^{\prime}$ is identified with the set of leaves for all $i \in I$. For each $i \in I$, we glue the rooted tree $J_{i}^{\prime}$ to the rooted tree $I^{\prime}$ by identifying the root of $J_{i}^{\prime}$ with the leaf $i$ of $I^{\prime}$. We obtain a rooted tree $J^{\prime}$ whose set of leaves is identified with the disjoint union of the sets $J_{i}, i \in I$. For each $i \in I$, $U_{i}=V_{i r_{i}}$, where $r_{i}$ is the root of $J_{i}^{\prime}$; hence we obtain a covering $\left(V_{j}\right)_{j \in J^{\prime}}$ such that the given rooted tree structure on $J^{\prime}$ is suitable for $\left(V_{j}\right)_{j \in J^{\prime}}$; indeed, this can be 
checked locally on the rooted tree $J^{\prime}$. We conclude that the composite covering $\left(V_{i j}\right)_{i \in I, j \in J_{i}}$ of $X$ is leaflike. The statement for $\mathscr{T}_{\text {aux }}$-admissible coverings now follows by passing to leaflike refinements.

Combining Lemma 2.32 and the fact that $\mathscr{T}_{\text {aux }}$-admissible coverings are stable under pullback, we see that the semiaffinoid subdomains and the $\mathscr{T}_{\text {aux }}$-admissible coverings define a G-topology on the category of semiaffinoid $K$-spaces equipped with the physical points functor. The following proposition suggests that $\mathscr{T}_{\text {aux }}$ should be viewed as an analog of the weak G-topology in rigid geometry. We first define:

Definition 2.33. A retrocompact covering of a semiaffinoid $K$-space $X$ is a finite family of retrocompact semiaffinoid subdomains of $X$ that covers $X$ on the level of physical points.

If $I$ is a rooted tree, we write $\operatorname{lv}(I)$ to denote the set of leaves of that tree, and we write $v(I)$ denote the volume of the tree, that is, its number of vertices.

Proposition 2.34. Retrocompact coverings of semiaffinoid spaces are $\mathscr{T}_{\text {aux }}$-admissible.

Proof. Let $X$ be a semiaffinoid $K$-space, and let $\left(X_{i}\right)_{i \in I}$ be a finite family of retrocompact semiaffinoid subdomains in $X$ covering $X$ on the level of sets; we have to show that $\left(X_{i}\right)_{i \in I}$ is $\mathscr{T}_{\text {aux }}$-admissible. For each $i \in I$, we choose a retrocompact representation $\varphi_{i}$ of $X_{i}$ in $X$, such that the targets of the $\varphi_{i}$ all coincide with a fixed flat affine target $\mathfrak{X}$. For each $i \in I$, we choose a factorization

$$
\varphi_{i}=\beta_{i 1} \circ \psi_{i 1} \circ \cdots \circ \beta_{i n_{i}} \circ \psi_{i n_{i}},
$$

where the $\psi_{i j}$ are open immersions and the $\beta_{i j}$ are admissible blowups,

$$
\mathfrak{X}_{i j} \stackrel{\psi_{i j}}{\rightarrow} \mathfrak{X}_{i j}^{\prime} \stackrel{\beta_{i j}}{\rightarrow} \mathfrak{X}_{i, j-1},
$$

with $\mathfrak{X}_{i 0}=\mathfrak{X}$. Let $v$ denote the sum of the $n_{i}$; we say that $v$ is the total length of the given retrocompact representation. Let $\mathfrak{X}^{\prime} \rightarrow \mathfrak{X}$ be an admissible blowup dominating all $\beta_{i 1}: \mathfrak{X}_{i 1}^{\prime} \rightarrow \mathfrak{X}$, and let $\mathfrak{U}_{i} \subseteq \mathfrak{X}^{\prime}$ denote the preimage of $\mathfrak{X}_{i 1} \subseteq \mathfrak{X}_{i 1}^{\prime}$. The $\mathfrak{X}_{i 1}^{\text {rig }}$ cover $\mathfrak{X}^{\text {rig }}$, the specialization map $\mathrm{sp}_{\mathfrak{X}^{\prime}}$ is surjective onto the closed points of $\mathfrak{X}^{\prime}$, and the closed points in $\mathfrak{X}^{\prime}$ lie very dense; hence $\mathfrak{X}^{\prime}$ is covered by the $\mathfrak{U}_{i}$. For each $i \in I$, we consider the pullback $\psi_{i}^{\prime}$ of

$$
\beta_{i 2} \circ \psi_{i 2} \circ \cdots \circ \beta_{i n_{i}} \circ \psi_{i n_{i}}
$$

under $\mathfrak{U}_{i} \subseteq \mathfrak{X}^{\prime} \rightarrow \mathfrak{X}_{i 1}^{\prime}$, and moreover for each $j \in I$ different from $i$ we consider the pullback $\varphi_{i j}^{\prime}$ of

$$
\varphi_{j}=\beta_{j 1} \circ \psi_{j 1} \circ \cdots \circ \beta_{j n_{j}} \circ \psi_{j n_{j}}
$$


under $\mathfrak{U}_{i} \subseteq \mathfrak{X}^{\prime} \rightarrow \mathfrak{X}$, both in the category of flat formal $R$-schemes of ff type. For each $i \in I$, we choose a finite affine covering of $\mathfrak{U}_{i}$. For each constituent $\mathfrak{V}_{i s}$ of this covering with semiaffinoid generic fiber $V_{i s}$, we choose finite affine coverings of $\left(\psi_{i}^{\prime}\right)^{-1}\left(\mathfrak{V}_{i s}\right)$ and of $\left(\varphi_{i j}^{\prime}\right)^{-1}\left(\mathfrak{V}_{i s}\right)$, for $j \in I \backslash\{i\}$. We obtain a retrocompact covering of $V_{i s}$, together with retrocompact representations as above of total length $v-1$. If we let $i$ and $s$ vary, the resulting retrocompact covering of $X$ refines $\left(X_{i}\right)_{i \in I}$. Since the $V_{i s}$, for varying $i$ and $s$, form an elementary covering of $X$, it suffices to see that the given retrocompact covering of $V_{i s}$ is $\mathscr{T}_{\text {aux }}$-admissible, which now follows by induction on $v$; the case $v=1$ is trivial.

Definition 2.35. Let $\mathscr{T}_{\text {urig }}$ denote the finest G-topology on the category of semiaffinoid $K$-spaces which is slightly finer than $\mathscr{T}_{\text {aux }}$ in the sense of [Bosch et al. 1984, 9.1.2/1].

The G-topology $\mathscr{T}_{\text {urig }}$ is called the uniformly rigid G-topology. It exists by [Bosch et al. 1984, 9.2.1/2], and it is saturated in the sense that it satisfies conditions $\left(\mathrm{G}_{0}\right)-\left(\mathrm{G}_{2}\right)$ in [Bosch et al. $1984,9.1 .2$ ], saying that $\mathscr{T}_{\text {urig }}$-admissibility of subsets can be checked locally with respect to $\mathscr{T}_{\text {urig-admissible coverings and that admis- }}$

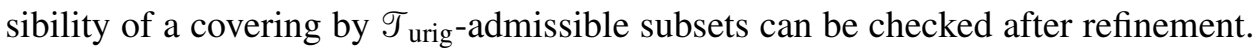

As a corollary of [BGR] 9.1.2/3, we obtain the following explicit description of the uniformly rigid G-topology on a semiaffinoid $K$-space:

Proposition 2.36. Let $X$ be a semiaffinoid $K$-space.

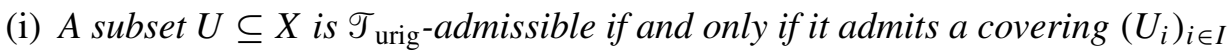
by semiaffinoid subdomains such that for any morphism $\varphi: Y \rightarrow X$ of semiaffinoid $K$-spaces with $\varphi(Y) \subseteq U$, the induced covering of $Y$ has a leaflike refinement.

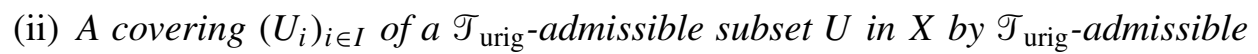

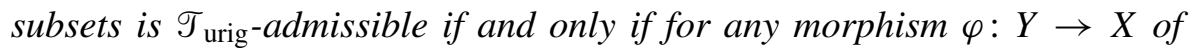
semiaffinoid $K$-spaces with $\varphi(Y) \subseteq U$, the induced covering of $Y$ has a leaflike refinement.

Corollary 2.37. Let $X$ be a semiaffinoid $K$-space.

(i) For any semiaffinoid subdomain $U$ of $X$, the uniformly rigid $G$-topology on $X$ restricts to the uniformly rigid $G$-topology on $U$.

(ii) If $U \subseteq X$ is a finite union of retrocompact semiaffinoid subdomains in $X$,

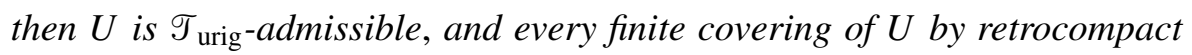
semiaffinoid subdomains in $X$ is $\mathscr{T}_{\text {urig-admissible. }}$

Proof. By Corollary 2.29(i), the semiaffinoid subdomains in $U$ are the semiaffinoid subdomains in $X$ contained in $U$, and by Corollary 2.25(iii) the semiaffinoid 
morphisms to $X$ with image in $U$ correspond to the semiaffinoid morphisms to $U$. Hence, statement (i) follows from parts (i) and (ii) of Proposition 2.36.

To prove the second statement, let $\left(U_{i}\right)_{i \in I}$ be a finite family of retrocompact semiaffinoid subdomains of $X$ such that $U$ is the union of the $U_{i}$. Let $Y$ be any semiaffinoid $K$-space, and let $\varphi: Y \rightarrow X$ be any semiaffinoid morphism whose image is lies in $U$. Then $\left(\varphi^{-1}\left(U_{i}\right)\right)_{i \in I}$ is a retrocompact covering of $Y$; by Proposition 2.34, it admits a leaflike refinement. By Proposition 2.36(i), we conclude that $U$ is a

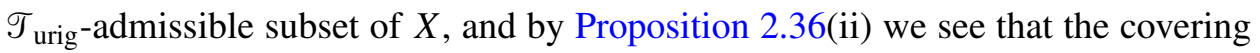
$\left(U_{i}\right)_{i \in I}$ of $U$ is $\mathscr{T}_{\text {urig-admissible. }}$

In particular, Corollary 2.37(ii) and the theorem of Gerritzen and Grauert [Bosch et al. 1984, 7.3.5/1] show that if $A$ is an affinoid $K$-algebra and if $U \subseteq \operatorname{Sp} A$ is an affinoid subdomain, then $U \subseteq \mathrm{sSp} A$ is $\mathscr{T}_{\text {urig-admissible. }}$

Remark 2.38 (quasicompactness). Proposition 2.36(ii) shows that semiaffinoid $K$ spaces are quasicompact in $\mathscr{T}_{\text {urig }}$, see [Bosch et al. 1984, p. 337]. By the maximum principle for affinoid $K$-algebras; it follows that $\mathrm{sSp}\left(R \llbracket S \rrbracket \otimes_{R} K\right)$ has no $\mathscr{T}_{\text {urig- }}$ admissible covering by semiaffinoid subdomains whose rings of functions are affinoid. In particular, the covering of $\mathrm{sSp}\left(R \llbracket S \rrbracket \otimes_{R} K\right)$ provided by Berthelot's construction is not $\mathscr{T}_{\text {urig-admissible. }}$

Remark 2.39 (bases for $\mathcal{T}_{\text {urig }}$ ). Proposition 2.36 implies that the semiaffinoid subdomains form a basis for the uniformly rigid G-topology on a semiaffinoid $K$ space [Bosch et al. 1984, p. 338]. The retrocompact semiaffinoid subdomains in sSp $(K\langle S\rangle)$ do not form a basis for $\mathscr{T}_{\text {urig }}$ : Indeed, $\mathrm{sSp}\left(R \llbracket S \rrbracket \otimes_{R} K\right)$ is a semiaffinoid subdomain in $\mathrm{sSp}(K\langle S\rangle)$; by Lemma 2.30 and Remark 2.38, it does not

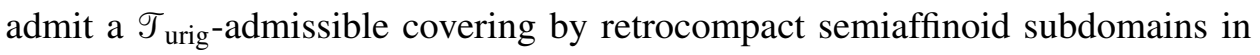
sSp $(K\langle S\rangle)$. Thus, even though the $K$-algebra $K\langle S\rangle$ is affinoid, the uniformly rigid G-topology on $\mathrm{sSp}(K\langle S\rangle)$ turns out to be strictly coarser than the rigid G-topology on $\operatorname{Sp}(K\langle S\rangle)$. We do not know whether this discrepancy already appears on the level of admissible subsets.

We conclude our discussion of the uniformly rigid G-topology $\mathscr{T}_{\text {urig }}$ by showing that it is finer than the Zariski topology $\mathscr{T}_{\text {Zar }}$ which, on a semiaffinoid $K$-space $X$, is generated by the nonvanishing loci $D(f)$ of semiaffinoid functions $f$ on $X$ :

Proposition 2.40. The uniformly rigid G-topology $\mathscr{T}_{\text {urig }}$ is finer than the Zariski topology $\mathscr{T}_{\mathrm{Zar}}$.

Proof. Let $X=\mathrm{sSp} A$ be a semiaffinoid $K$-space, let $U \subseteq X$ be a Zariski-open subset, and let $f_{1}, \ldots, f_{n} \in A$ be semiaffinoid functions such that $U$ is the union of the Zariski-open subsets $D\left(f_{i}\right)=\left\{x \in \operatorname{Max} A ; f_{i}(x) \neq 0\right\}$. Let $Y$ be a nonempty semiaffinoid $K$-space, and let $\varphi: Y \rightarrow X$ be a semiaffinoid morphism whose image is contained in $U$. For each $i$, the preimage $\varphi^{-1}\left(D\left(f_{i}\right)\right)$ is the set of points $y \in Y$ 
where $\varphi^{*} f_{i} \neq 0$. Since $Y$ is covered by the $\varphi^{-1}\left(D\left(f_{i}\right)\right)$, the $\varphi^{*} f_{i}$ generate the unit ideal in $B$. That is, there exist elements $b_{1}, \ldots, b_{n}$ in $B$ such that $b_{1} \varphi^{*} f_{1}+\ldots+$ $b_{n} \varphi^{*} f_{n}=1$. Let us set $\gamma:=\left(\max _{i}\left|b_{i}\right|_{\text {sup }}\right)^{-1}$; this number is well-defined since the $b_{i}$ are bounded functions on $Y$ without a common zero. By the strict triangle inequality, $\max _{i}\left|\varphi^{*} f_{i}(y)\right| \geq \gamma$ for all $y \in Y$. For each $i$, let $Y_{i} \subseteq Y$ denote the set of points $y \in Y$ where $\left|\varphi^{*} f_{i}(y)\right| \geq \gamma$; then $\left(Y_{i}\right)_{1 \leq i \leq n}$ is a retrocompact covering of $Y$ refining $\left(\varphi^{-1}\left(D\left(f_{i}\right)\right)\right)_{1 \leq i \leq n}$. By Proposition 2.34, retrocompact coverings are

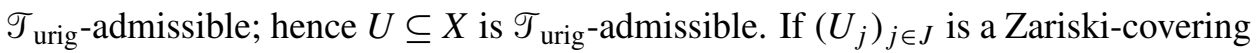
of $U$, we may pass to a refinement and assume that for all $j \in J, U_{j}=D\left(g_{j}\right) \subseteq X$ for some semiaffinoid function $g_{j}$ on $X$; we can then argue along the same lines

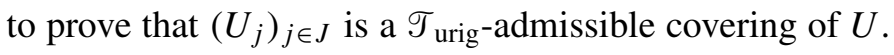

The above argument works even though the maximum principle might fail on $Y$. Let us point out that our proof shows the following: If $f_{1}, \ldots, f_{n}$ are semiaffinoid functions on $X$, if

$$
U=\bigcup_{i=1}^{n} D\left(f_{i}\right)
$$

is the associated Zariski-open subset of $X$, and if we set

$$
U_{\geq \varepsilon}=\bigcup_{i=1}^{n}\left\{x \in X ;\left|f_{i}(x)\right| \geq \varepsilon\right\}
$$

for $\varepsilon \in \sqrt{\left|K^{*}\right|}$, then the resulting covering $\left(U_{\geq \varepsilon}\right)_{\varepsilon}$ of $U$ by finite unions of retrocompact semiaffinoid subdomains of $X$ is $\mathscr{T}_{\text {urig }}$-admissible. In particular, Zariskiopen subsets in semiaffinoid spaces need not be quasicompact in the uniformly rigid G-topology. As a consequence, the sheaf of uniformly rigid functions on a semiaffinoid $K$-space, to be defined in the following section, may have unbounded sections on Zariski-open subsets.

2C4. The acyclicity theorem. Let $X$ be a semiaffinoid $K$-space. We show that the presheaf $O_{X}$ that we introduced after Definition 2.20 is a sheaf for $\mathscr{T}_{\text {aux }}$ and, hence, extends uniquely to a sheaf for $\mathcal{T}_{\text {urig. }}$. More generally, we show that every $\mathrm{O}_{X}$-module associated to a finite module over the ring of global functions on $X$ is

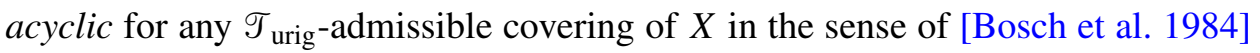
p. 324. Adopting methods from [Lütkebohmert 1990], we derive our acyclicity theorem from results in formal geometry; we also use ideas from [Lipshitz and Robinson 2000, III.3.2].

Let us recall from [Bosch et al. 1984, p. 324] that if $\mathscr{F}$ is a presheaf in $\mathrm{O}_{X^{-}}$ modules on $\mathscr{T}_{\text {aux }}$, a covering $\left(X_{i}\right)_{i \in I}$ of $X$ by semiaffinoid subdomains is called $\mathscr{F}_{-}$ acyclic if the associated augmented Čech complex is acyclic. The covering $\left(X_{i}\right)_{i \in I}$ 
is called universally $\mathscr{F}$-acyclic if $\left(X_{i} \cap U\right)_{i \in I}$ is $\left.\mathscr{F}\right|_{U}$-acyclic for any semiaffinoid subdomain $U \subseteq X$.

Theorem 2.41. For a semiaffinoid $K$-space $X, \mathscr{T}_{\text {aux }}$-admissible coverings are $\mathcal{O}_{X}$ acyclic.

Proof. Let us first consider an elementary covering $\left(X_{i}\right)_{i \in I}$. Let us choose a formal representation $\left(\mathfrak{X}, \beta: \mathfrak{X}^{\prime} \rightarrow \mathfrak{X},\left(\mathfrak{X}_{i}\right)_{i \in I}\right)$ of $\left(X_{i}\right)_{i \in I}$, where $\beta$ is an admissible blowup and where $\left(\mathfrak{X}_{i}\right)_{i \in I}$ is a finite affine covering of $\mathfrak{X}^{\prime}$ such that $\mathfrak{X}_{i} \subseteq \mathfrak{X}^{\prime} \rightarrow \mathfrak{X}$ represents $X_{i}$ in $X$. By the ff type transcription of [Lütkebohmert 1990, 2.1], $\beta^{\sharp} \otimes_{R} K$ is an isomorphism; hence $\beta$ induces a natural identification of augmented Čech complexes

$$
C_{\text {aug }}^{\bullet}\left(\left(X_{i}\right)_{i \in I}, \mathscr{O}_{X}\right) \cong C_{\text {aug }}^{\bullet}\left(\left(\mathfrak{X}_{i}\right)_{i \in I}, \mathcal{O}_{\mathfrak{X}^{\prime}} \otimes_{R} K\right) .
$$

We have to show that the complex on the right hand side is acyclic. Since $0_{\mathfrak{X}^{\prime}} \otimes_{R} K$ is a sheaf on $\mathfrak{X}^{\prime}$, it suffices to show that

$$
\check{H}^{q}\left(\left(\mathfrak{X}_{i}\right)_{i \in I}, \mathcal{O}_{\mathfrak{X}^{\prime}} \otimes_{R} K\right)=0
$$

for all $q \geq 1$. Since $I$ is finite, we have an identification

$$
\check{H}^{q}\left(\left(\mathfrak{X}_{i}\right)_{i \in I}, \mathcal{O}_{\mathfrak{X}^{\prime}} \otimes_{R} K\right)=\check{H}^{q}\left(\left(\mathfrak{X}_{i}\right)_{i \in I}, \mathcal{O}_{\mathfrak{X}^{\prime}}\right) \otimes_{R} K .
$$

By the comparison theorem [Grothendieck 1961b, 4.1.5 and 4.1.7] and by the vanishing theorem [Grothendieck 1961b, 1.3.1], the higher cohomology groups of a coherent sheaf on an affine noetherian formal scheme vanish. Since the $\mathfrak{X}_{i}$ are affine, Leray's theorem implies that

$$
\check{H}^{q}\left(\left(\mathfrak{X}_{i}\right)_{i \in I}, \mathcal{O}_{\mathfrak{X}^{\prime}}\right)=H^{q}\left(\mathfrak{X}^{\prime}, \mathcal{O}_{\mathfrak{X}^{\prime}}\right) .
$$

By [Grothendieck 1961b, 1.4.11], $H^{q}\left(\mathfrak{X}^{\prime}, \mathcal{O}_{\mathfrak{X}^{\prime}}\right)=\Gamma\left(\mathfrak{X}, R^{q} \beta_{*} \mathcal{O}_{\mathfrak{X}^{\prime}}\right)$, and by the ff type transcription of [Lütkebohmert 1990, 2.1] this module is $\pi$-torsion. We have thus finished the proof in the case where $\left(X_{i}\right)_{i \in I}$ is an elementary covering.

Let us turn towards the general case. By definition, every $\mathcal{T}_{\text {aux }}$-admissible covering of $X$ has a leaflike refinement; by [Bosch et al. 1984, 8.1.4/3] it is enough to show that the leaflike coverings of $X$ are universally $O_{X}$-acyclic. Since leaflike coverings are preserved with respect to pullback under morphisms of semiaffinoid $K$-spaces, it suffices to show that any leaflike covering $\left(X_{i}\right)_{i \in I}$ of $X$ is $\mathscr{O}_{X}$-acyclic.

Let $\left(X_{j}\right)_{j \in J}$ be a treelike covering of $X$ extending $\left(X_{i}\right)_{i \in I}$, and let us choose a suitable rooted tree structure on $J$ such that $I \subseteq J$ is identified with the set of leaves of $J$. We argue by induction on the volume of $J$. If $J$ has only one vertex, the covering $\left(X_{i}\right)_{i \in I}$ is trivial and, hence, $O_{X}$-acyclic. Let us assume that $J$ has more than one vertex. Let $\iota \in I$ be a leaf of $J$ such that the length $l(\iota)$ of the path from $\iota$ to the root is maximal in $\{l(i) ; i \in I\}$. Let $\iota^{\prime}:=\operatorname{par}(\iota)$ denote the parent of $\iota$. 
By maximality of $l(\iota)$, all siblings $i \in \operatorname{ch}\left(\iota^{\prime}\right)$ of $\iota$ are leaves of $J$. Let $J^{\prime}:=J \backslash \operatorname{ch}\left(\iota^{\prime}\right)$ be the rooted subtree of $J$ that is obtained by removing the siblings of $\iota$ (including $\iota$ itself). Then

(i) the set of leaves of $J^{\prime}$ is $I^{\prime}:=\left(I \backslash \operatorname{ch}\left(\iota^{\prime}\right)\right) \cup\left\{\iota^{\prime}\right\}$,

(ii) $\left(X_{j}\right)_{j \in J^{\prime}}$ is a treelike covering of $X$, and

(iii) $v\left(J^{\prime}\right)<v(J)$.

By our induction hypothesis, the covering $\left(X_{i}\right)_{i \in I^{\prime}}$ is $\mathbb{O}_{X}$-acyclic. Since $\left(X_{i}\right)_{i \in I}$ is a refinement of $\left(X_{i}\right)_{i \in I^{\prime}}$, [Bosch et al. 1984, 8.1.4/3] says that it suffices to prove that for any $r \geq 0$ and any tuple $\left(i_{0}, \ldots, i_{r}\right) \in\left(I^{\prime}\right)^{r+1}$, the covering $\left(X_{i} \cap X_{i_{0} \cdots i_{r}}\right)_{i \in I}$ of $X_{i_{0} \cdots i_{r}}$ is $\mathrm{O}_{X}$-acyclic, where $X_{i_{0} \cdots i_{r}}$ denotes the intersection $X_{i_{0}} \cap \ldots \cap X_{i_{r}}$. Let us assume that there exists some $0 \leq s \leq r$ such that $i_{s} \neq \iota^{\prime}$. Then $i_{s} \in I$. Since $X_{i_{0} \cdots i_{r}} \subseteq X_{i_{s}}$, we see that the trivial covering of $X_{i_{0} \cdots i_{r}}$ refines $\left(X_{i} \cap X_{i_{0} \cdots i_{r}}\right)_{i \in I}$. Since trivial coverings restrict to trivial coverings and since trivial coverings are acyclic, we deduce from [Bosch et al. 1984, 8.1.4/3] that $\left(X_{i} \cap X_{i_{0} \cdots i_{r}}\right)_{i \in I}$ is acyclic. It remains to consider the case where all $i_{s}, 0 \leq s \leq r$, coincide with $\iota^{\prime}$. That is, it remains to see that the covering $\left(X_{i} \cap X_{\iota^{\prime}}\right)_{i \in I}$ of $X_{\iota^{\prime}}$ is $\mathcal{O}_{X}$-acyclic. It admits the elementary covering $\left(X_{i}\right)_{i \in \operatorname{ch}\left(\iota^{\prime}\right)}$ as a refinement. Since elementary coverings restrict to elementary coverings and since elementary coverings are ${ } X_{X}$-acyclic by what we have shown so far, we conclude by [Bosch et al. 1984, 8.1.4/3] that $\left(X_{i} \cap X_{\iota^{\prime}}\right)_{i \in I}$ is $O_{X}$-acyclic, as desired.

By [Bosch et al. 1984, 9.2.3/1], $\mathscr{O}_{X}$ extends uniquely to a sheaf for $\mathscr{T}_{\text {urig }}$ which we again denote by $\mathrm{O}_{X}$ and which we call the structural sheaf or the sheaf of uniformly rigid functions. We can now easily discuss a fundamental example of a nonadmissible finite covering of a semiaffinoid $K$-space by semiaffinoid subdomains:

Example 2.42. The canonical covering of the semiaffinoid closed unit disc

$$
\mathrm{sSp}(K\langle T\rangle)
$$

by the semiaffinoid open unit disc $\operatorname{sSp}\left(R \llbracket T \rrbracket \otimes_{R} K\right)$ and the semiaffinoid unit circle

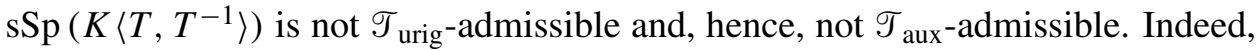
the two covering sets are nonempty and disjoint, while the ring of functions $K\langle T\rangle$ on the closed semiaffinoid unit disc has no nontrivial idempotents.

If $X$ is a semiaffinoid $K$-space with ring of global functions $A$ and if $M$ is a finite $A$-module, the presheaf $M \otimes 0_{X}$ sending a semiaffinoid subdomain $U$ in $X$ to $M \otimes_{A} \mathrm{O}_{X}(U)$ is an $\mathrm{O}_{X}$-module, which we call the $\mathrm{O}_{X}$-module associated to $M$. A presheaf $\mathscr{F}$ equipped with an $\mathbb{O}_{X}$-module structure is called associated if it is isomorphic to $M \otimes \mathrm{O}_{X}$ for some finite $A$-module $M$. We sometimes abbreviate $\tilde{M}:=M \otimes \mathbb{O}_{X}$. 
Corollary 2.43. Let $X$ be a semiaffinoid $K$-space, and let $\mathscr{F}$ be an associated $\mathrm{O}_{X^{-}}$ module. Then every $\mathcal{T}_{\text {aux }}$-admissible covering $\left(X_{i}\right)_{i \in I}$ of $X$ is $\mathscr{F}_{\text {-acyclic. }}$

Proof. By [Bosch et al. 1984, 8.1.4/3], we may assume that $I$ is finite. Using Theorem 2.41, the proof is now literally the same as the proof of [Bosch et al. $1984,8.2 .1 / 5]$.

In particular, $M \otimes \mathscr{O}_{X}$ is a $\mathscr{T}_{\text {aux }}$-sheaf. By [Bosch et al. 1984, 9.2.3/1], $M \otimes \mathscr{O}_{X}$

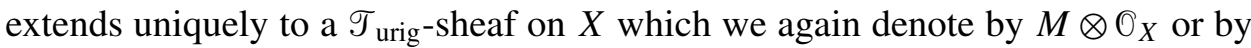
$\tilde{M}$ and which we call the sheaf associated to $M$.

Remark 2.44. If $U \subseteq X$ is a representable subset that is $\mathscr{T}_{\text {urig-admissible, then }}$ $\mathrm{O}_{X}(U)=\mathscr{O}_{U}(U)$. Indeed, $U$ admits a $\mathscr{T}_{\text {urig, } X \text {-admissible covering by semiaffinoid }}$ subdomains in $X$; since morphisms of semiaffinoid spaces are continuous for $\mathscr{T}_{\text {urig }}$, this covering is also $\mathscr{T}_{\text {urig, } U \text {-admissible, so the statement follows from the fact that }}$ both $O_{X}$ and $O_{U}$ are $\mathscr{T}_{\text {urig }}$-sheaves. However, it is not clear whether $\mathscr{T}_{\text {urig, } X}$ restricts to $\mathscr{T}_{\text {urig, } U}$; for example, we do not know whether a semiaffinoid subdomain of $U$ is $\mathscr{T}_{\text {urig, } X \text {-admissible. Of course, this does not affect our theory since we do not }}$ deal with general representable subsets.

The category of abelian sheaves on $\left(X,\left.\mathscr{T}_{\text {urig }}\right|_{X}\right)$ has enough injective objects, so the functor $\Gamma(X, \cdot)$ from the category of abelian sheaves on $X$ to the category of abelian groups has a right derived functor $H^{\bullet}(X, \cdot)$. By the acyclicity theorem and its Corollary 2.43, this right derived functor can, for associated $\mathrm{O}_{X}$-modules, be calculated in terms of Čech cohomology:

Corollary 2.45. Let $X$ be a semiaffinoid $K$-space, and let $\mathscr{F}$ be an associated $\mathcal{O}_{X}$-module. Then the natural homomorphism $\check{H}^{q}(U, \mathscr{F}) \rightarrow H^{q}(U, \mathscr{F})$ is an iso-

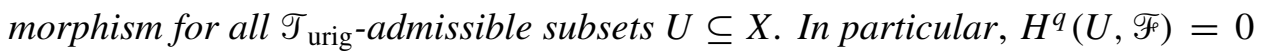
for all $q>0$ and all semiaffinoid subdomains $U \subseteq X$.

Proof. The system $S$ of semiaffinoid subdomains in $X$ satisfies the following properties:

(i) $S$ is stable under the formation of intersections,

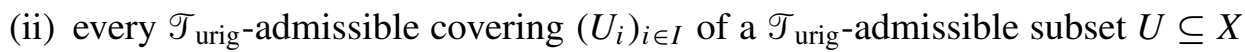
admits a $\mathscr{T}_{\text {urig-admissible refinement by sets in } S \text {, and }}$

(iii) $\check{H}^{q}(U, \mathscr{F})$ vanishes for all $q>0$ and all $U \in S$;

hence the statement follows by means of the standard Čech spectral sequence argument.

Transcribing the proof of [Bosch et al. 1984, 7.3.2/1], we see that if $A$ is a semiaffinoid $K$-algebra with associated semiaffinoid $K$-space $X$ and if $\mathfrak{m} \subseteq A$ is a maximal ideal corresponding to a point $x \in X$, then the stalk $O_{X, x}$ is local with 
maximal ideal $\mathfrak{m} \mathfrak{O}_{X, x}$ which coincides with the ideal of germs of functions vanishing in $x$. The arguments in the proof of [ibid., 7.3.2/3] are also seen to work in our situation, showing that the natural homomorphisms $A / \mathfrak{m}^{n+1} \rightarrow \mathrm{O}_{X, x} / \mathfrak{m}^{n+1} \mathrm{O}_{X, x}$ are isomorphisms for all $n \in \mathbb{N}$. The rings $O_{X, x}$ are noetherian, which can for example be seen by imitating the proof of [ibid., 7.3.2/7].

Transcribing the discussion at the beginning of [ibid., 9.3.1], we see that the uniformly rigid G-topology and the sheaf of uniformly rigid functions define a functor from the category of semiaffinoid $K$-spaces to the category of locally ringed G-topological $K$-spaces. The proof of [ibid., 9.3.1/2] carries over verbatim to the semiaffinoid situation, showing that this functor is fully faithful. We call a locally ringed G-topological $K$-space semiaffinoid if it lies in the essential image of this functor.

2D. Uniformly rigid spaces. We are now able to define the category of uniformly rigid $K$-spaces:

Definition 2.46. Let $X$ be a locally ringed G-topological $K$-space.

(i) An admissible semiaffinoid covering of $X$ is an admissible covering $\left(X_{i}\right)_{i \in I}$ of $X$ such that for each $i \in I,\left(X_{i},\left.O_{X}\right|_{X_{i}}\right)$ is a semiaffinoid $K$-space.

(ii) The space $X$ is called uniformly rigid if it satisfies conditions $\left(\mathrm{G}_{0}\right)-\left(\mathrm{G}_{1}\right)$ in [Bosch et al. 1984, 9.1.2] and if it admits an admissible semiaffinoid covering.

(iii) An admissible open subset $U$ of a uniformly rigid $K$-space $X$ is called an open semiaffinoid subspace of $X$ if $\left(U,\left.O_{X}\right|_{U}\right)$ is a semiaffinoid $K$-space.

Remark 2.47. In the author's $\mathrm{PhD}$ thesis, open semiaffinoid subspaces were simply called semiaffinoid subspaces [Kappen 2009, Section 1.3.9]

The category $\mathrm{uRig}_{K}$ of uniformly rigid $K$-spaces is a full subcategory of the category of locally G-topological $K$-spaces, and it contains the category of semiaffinoid $K$-spaces as a full subcategory.

Remark 2.48. We do not know whether an open semiaffinoid subspace $U$ of a semiaffinoid $K$-space $X$ is necessarily a semiaffinoid subdomain in $X$. However, one easily verifies that $U$ is a representable subset in $X$. Moreover, one sees that $U$ is locally a semiaffinoid subdomain in $X$; see Lemma 2.52 for a precise statement. In rigid geometry, the open affinoid subvarieties [Bosch et al. 1984, p. 357] of an affinoid space are precisely the affinoid subdomains, which means that there is no need to distinguish between the two notions in the affinoid setting.

Remark 2.49. Let $X=\operatorname{sSp} A$ be a semiaffinoid $K$-space, and let $U=\operatorname{sSp} B$ be an open semiaffinoid subspace of $X$; then the restriction homomorphism $A \rightarrow B$ is flat. Indeed, for every maximal ideal $\mathfrak{n} \subseteq B$ with corresponding point $x \in U$ and preimage $\mathfrak{m} \subseteq A$, the induced homomorphism $A_{\mathfrak{m}} \rightarrow B_{\mathfrak{n}}$ induces an isomorphism of 
maximal-adic completions; by the flatness criterion [Bourbaki 1998, III.5.2, Theorem 1], we conclude that $A \rightarrow B_{\mathfrak{n}}$ is flat for all maximal ideals $\mathfrak{n}$ in $B$, which implies that $A \rightarrow B$ is flat.

Lemma 2.50. The open semiaffinoid subspaces of a uniformly rigid $K$-space $X$ form a basis for the G-topology on X.

Proof. Let $\left(X_{i}\right)_{i \in I}$ be an admissible semiaffinoid covering of $X$, and let $U \subseteq X$ be an admissible open subset. Then $\left(X_{i} \cap U\right)_{i \in I}$ is an admissible covering of $U$. For each $i \in I, X_{i} \cap U$ is admissible open in $X_{i}$ and, hence, admits an admissible covering by semiaffinoid subdomains of $X_{i}$. Hence, $U$ has an admissible semiaffinoid covering.

It follows that if $X$ is a uniformly rigid $K$-space and if $U \subseteq X$ is an admissible open subset, then $\left(U,\left.O_{X}\right|_{U}\right)$ is a uniformly rigid $K$-space, again.

It is now clear that the gluing theorem [Bosch et al. 1984, 9.3.2/1] and its proof carry over verbatim to the uniformly rigid setting. Similarly, a morphism of uniformly rigid spaces can be defined locally on the domain; this is the uniformly rigid version of [ibid., 9.3.3/1], and again the proof is obtained by literal transcription. Furthermore, a uniformly rigid $K$-space is determined by its functorial points with values in semiaffinoid $K$-spaces.

We can also copy the proof of [ibid., 9.3.3/2] to see that if $X$ is a semiaffinoid $K$-space and if $Y$ is a uniformly rigid $K$-space, then the set of morphisms from $Y$ to $X$ is naturally identified with the set of $K$-algebra homomorphisms from $0_{X}(X)$ to $\mathscr{O}_{Y}(Y)$.

Let $\mathfrak{X}$ be an affine formal $R$-scheme of ff type with semiaffinoid generic fiber $X$. The associated specialization map $\mathrm{sp}_{\mathfrak{X}}$ which we discussed in Section $2 \mathrm{~B} 1$ is naturally enhanced to a morphism of G-ringed $R$-spaces $\mathrm{sp}_{\mathfrak{X}}: X \rightarrow \mathfrak{X}$. Morphisms of uniformly rigid $K$-spaces being defined locally on the domain, we see that $\mathrm{sp}_{\mathfrak{X}}$ is final among all morphisms of G-ringed $R$-spaces from uniformly rigid $K$-spaces to $\mathfrak{X}$. Using this universal property, we can invoke gluing techniques to construct the uniformly rigid generic fiber $\mathfrak{X}^{\text {urig }}$ of a general formal $R$-scheme of locally ff type $\mathfrak{X}$, together with a functorial specialization map $\mathrm{sp}_{\mathfrak{X}}: \mathfrak{X}^{\text {urig }} \rightarrow \mathfrak{X}$ which is universal among all morphisms of G-ringed $R$-spaces from uniformly rigid $K$-spaces to $\mathfrak{X}$; this process does not involve Berthelot's construction. It is easily seen that urig is faithful on the category of flat formal $R$-schemes of locally ff type. A formal $R$-model of a uniformly rigid $K$-space $X$ is a formal $R$-scheme $\mathfrak{X}$ of locally ff type together with an isomorphism $X \cong \mathfrak{X}^{\text {urig }}$. The map $\mathrm{sp}_{\mathfrak{X}}$ is surjective onto the closed points of $\mathfrak{X}$ whenever $\mathfrak{X}$ is flat over $R$. This follows from Remark 2.5, together with the remark that the underlying topological space of $\mathfrak{X}$ is a Jacobson space [Grothendieck and Dieudonné 1971, 0.2.8 and 6.4], so that the condition on a point in $\mathfrak{X}$ of being closed is local. 
Question 2.51. Under what conditions does a uniformly rigid $K$-space admit a formal $R$-model?

By Proposition 2.16, the category of semiaffinoid $K$-spaces has fibered products; following the method outlined in [Bosch et al. 1984, 9.3.5], we see that the category of uniformly rigid $K$-spaces has fibered products as well and that these are constructed by gluing semiaffinoid fibered products of open semiaffinoid subspaces. It is clear from this description that the urig-functor preserves fibered products.

Open semiaffinoid subspaces of semiaffinoid spaces can be described in the style of the Gerritzen-Grauert theorem [ibid., 7.3.5/3]:

Lemma 2.52. Let $X$ be a semiaffinoid $K$-space, and let $U \subseteq X$ be an open semiaffinoid subspace. Then $U$ admits a leaflike covering $\left(U_{i}\right)_{i \in I}$ such that each $U_{i}$ is a semiaffinoid subdomain in $X$.

Proof. By Lemma 2.50, $U$ admits an admissible covering $\left(V_{j}\right)_{j \in J}$ by semiaffinoid subdomains $V_{j}$ of $X$; by Proposition 2.36, this covering is refined by a leaflike covering $\left(U_{i}\right)_{i \in I}$ of $U$. Via pullback, the $V_{j}$ are semiaffinoid subdomains of $U$. Let $\varphi: I \rightarrow J$ denote a refinement map. By Corollary 2.29(i), for each $i \in I$ the set $U_{i}$ is a semiaffinoid subdomain in $V_{\varphi(i)}$ and, hence, in $X$, as desired.

A morphism of uniformly rigid $K$-spaces is called flat in a point of its domain if it induces a flat homomorphism of stalks in this point, and it is called flat if it is flat in all points. Clearly a morphism of semiaffinoid $K$-spaces is flat in this sense if and only if the underlying homomorphism of rings of global sections is flat.

2D1. Comparison with rigid geometry. In Section $2 \mathrm{C} 1$, we have defined the rigid space $X^{\mathrm{r}}$ associated to a semiaffinoid $K$-space $X=\mathrm{sSp} A$ together with a universal $K$-homomorphism $A \rightarrow \Gamma\left(X^{\mathrm{r}}, \mathrm{O}_{X^{\mathrm{r}}}\right)$ which induces a bijection $X^{\mathrm{r}} \rightarrow X$ of physical points and isomorphisms of completed stalks. We will show that this universal homomorphism extends to a morphism $\operatorname{comp}_{X}: X^{\mathrm{r}} \rightarrow X$ of locally G-ringed $K$ spaces which is final among all morphisms from rigid $K$-spaces to $X$. To do so, we first show that the above bijection is continuous, that is, that the rigid G-topology $\mathscr{T}_{\text {rig }}$ is finer than $\mathscr{T}_{\text {urig. }}$. We will need the following elementary fact from rigid geometry; the proof is left as an exercise to the reader:

Lemma 2.53. Let $X$ be an affinoid $K$-space, and let $U \subseteq X$ be a subset admitting a covering $\left(U_{i}\right)_{i \in I}$ by admissible open subsets $U_{i} \subseteq X$ such that for any affinoid $K$-space $Y$ and any morphism $\varphi: Y \rightarrow X$ with image in $U$, the induced covering $\left(\varphi^{-1}\left(U_{i}\right)\right)_{i \in I}$ of $Y$ has a refinement which is a finite covering by affinoid subdomains. Then $U \subseteq X$ is admissible.

Proposition 2.54. The rigid G-topology $\mathscr{T}_{\text {rig }}$ on $X$ is finer than the uniformly rigid G-topology $\mathscr{T}_{\text {urig. }}$ 
Proof. It is clear that $\mathscr{T}_{\text {aux }}$-admissible subsets and $\mathscr{T}_{\text {aux }}$-admissible coverings are

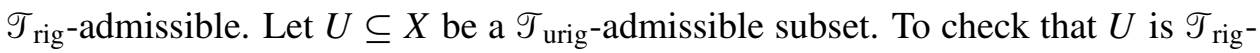
admissible, we may work locally on $X^{\mathrm{r}}$. Let $V^{\prime} \subseteq X^{\mathrm{r}}$ be an affinoid subspace; by Proposition 2.18, the open immersion $V^{\prime} \hookrightarrow X^{\mathrm{r}}$ corresponds to a morphism $V \rightarrow X$, where $V$ denotes the semiaffinoid $K$-space associated to $V^{\prime}$ such that $V^{\prime}=V^{\mathrm{r}}$. After pulling $U$ back under this morphism, we may thus assume that the $K$-algebra of global functions on $X$ is affinoid. Let $\left(U_{i}\right)_{i \in I}$ be a covering of $U$ by semiaffinoid subdomains in $X$ such that condition (i) of Proposition 2.36 is satisfied. Let $Y$ be an affinoid $K$-space, and let $\varphi: Y \rightarrow X^{\mathrm{r}}$ be a morphism of rigid spaces that factors through $U$. By Proposition 2.18, we may also view $\varphi$ as a morphism of semiaffinoid $K$-spaces. By assumption, the covering $\left(\varphi^{-1}\left(U_{i}\right)\right)_{i \in I}$ of $Y$ has a leaflike refinement; by Lemma 2.30, this refinement is affinoid. It now follows from Lemma 2.53 that $U \subseteq X$ is $\mathscr{T}_{\text {rig-admissible. }}$

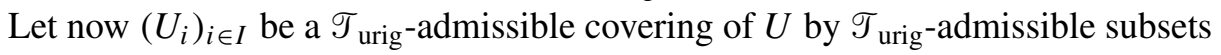
$U_{i}$. We have seen that $U$ and the $U_{i}$ are $\mathscr{T}_{\text {rig-admissible; we claim that the covering }}$ $\left(U_{i}\right)_{i \in I}$ is $\mathscr{T}_{\text {rig }}$-admissible as well. Again, we may work locally on $X^{\mathrm{r}}$ and thereby assume that the $K$-algebra of functions on $X$ is affinoid. Let $Y$ be an affinoid $K$ space, and let $\varphi: Y \rightarrow X^{\mathrm{r}}$ be a morphism of affinoid $K$-spaces, which we may also view as a morphism of semiaffinoid $K$-spaces. Since $\left(U_{i}\right)_{i \in I}$ is $\mathscr{T}_{\text {urig-admissible, }}$ we see by Proposition 2.36(ii) that $\left(\varphi^{-1}\left(U_{i}\right)\right)_{i \in I}$ has a leaflike and, hence, affinoid refinement. It follows that $\left(U_{i}\right)_{i \in I}$ is $\mathscr{T}_{\text {rig }}$-admissible.

If $U \subseteq X$ is a semiaffinoid subdomain, then the morphism $U^{\mathrm{r}} \rightarrow X^{\mathrm{r}}$ provided by Proposition 2.18 is an open immersion onto the preimage of $U$ under the continuous bijection $\operatorname{comp}_{X}: X^{\mathrm{r}} \rightarrow X$; hence $\operatorname{comp}_{X}$ extends to a morphism of G-ringed $K$ spaces with respect to $\mathscr{T}_{\text {aux }}$, which then again extends uniquely to a morphism of G-ringed $K$-spaces with respect to $\mathscr{T}_{\text {urig. }}$. One easily verifies that comp $\mathrm{p}_{X}$ is local.

Proposition 2.55. The morphism $\operatorname{comp}_{X}$ is final among all morphisms from rigid $K$-spaces to $X$.

Proof. Let $Y$ be a rigid $K$-space, and let $\psi: Y \rightarrow X$ be a morphism of locally G-ringed $K$-spaces. By Proposition 2.18, there is a unique morphism $\psi^{\mathrm{r}}: Y \rightarrow X^{\mathrm{r}}$ such that $\psi$ and $\operatorname{comp}_{X} \circ \psi^{\mathrm{r}}$ coincide on global sections. Since the points and the completed stalks of $X$ are recovered from the $K$-algebra of global sections of $X$, it follows that $\psi$ and $\operatorname{comp}_{X} \circ \psi^{\mathrm{r}}$ coincide.

Let $X$ be any uniformly rigid $K$-space. Since the open semiaffinoid subspaces of $X$ form a basis for the G-topology on $X$, we can use standard gluing arguments to show that the comparison morphisms attached to these open semiaffinoid subspaces glue to a universal comparison morphism

$$
\operatorname{comp}_{X}: X^{\mathrm{r}} \rightarrow X
$$


from a rigid $K$-space to $X$.

Remark 2.56. The functor $X \mapsto X^{\mathrm{r}}$ is faithful, yet not fully faithful. For example, it is easily seen that an unbounded function on the rigid open unit disc induces a morphism to the rigid projective line over $K$ which is not induced by a morphism from the semiaffinoid open unit disc $\operatorname{sSp}\left(R \llbracket S \rrbracket \otimes_{R} K\right)$ to the uniformly rigid projective line over $K$. Likewise, the functor $r$ forgets the distinction between the semiaffinoid open unit disc just mentioned and the uniformly rigid open unit disc that is the generic fiber of a quasiparacompact formal $R$-model of locally tf type for the rigid open unit disc. One can prove that $X \mapsto X^{\mathrm{r}}$ is fully faithful on the full subcategory of reduced semiaffinoid $K$-spaces.

Remark 2.57. The functor $X \mapsto X^{\mathrm{r}}$ preserves fibered products. Indeed, this may be checked in the semiaffinoid situation, where it follows from the fact that fibered products of semiaffinoid spaces are uniformly rigid generic fibers of fibered products of affine flat formal $R$-models, together with the fact that Berthelot's generic fiber functor preserves fibered products [de Jong 1995, 7.2.4(g)]. In particular, $X \mapsto X^{\mathrm{r}}$ preserves group structures.

Remark 2.58. We have seen that $\operatorname{comp}_{X}$ induces isomorphisms of completed stalks. Examining Berthelot's construction, one easily sees that $\operatorname{comp}_{X}$ in fact already induces isomorphisms of noncompleted stalks; the proof of this statement is left as an exercise.

We have seen that every uniformly rigid $K$-space $X$ has an underlying classical rigid $K$-space $X^{\mathrm{r}}$ such that $X$ and $X^{\mathrm{r}}$ share all local properties. That is, a uniformly rigid $K$-space can be seen as a rigid $K$-space equipped with an additional global uniform structure. Every quasiparacompact and quasiseparated rigid $K$-space carries a canonical uniformly rigid structure, which may be called the Raynaud-type uniform structure: let $\mathfrak{C}$ temporarily denote the category of quasiparacompact flat formal $R$-schemes of locally tf type, and let $\mathfrak{C}_{\mathrm{Bl}}$ denote its localization with respect to the class of admissible formal blowups. It follows easily from the definitions that the functor urig $\left.\right|_{\mathfrak{C}}: \mathfrak{C} \rightarrow \mathrm{uRig}_{K}$ factors through a functor $\mathrm{ur}^{\prime}: \mathfrak{C}_{\mathrm{Bl}} \rightarrow \mathrm{uRig}_{K}$. By [Bosch 2005, Theorem 2.8/3], the functor rig induces an equivalence $\operatorname{rig}_{\mathrm{Bl}}$ between $\mathfrak{C}_{\mathrm{B} 1}$ and the category $\mathrm{Rig}_{K}^{\prime}$ of quasiparacompact and quasiseparated rigid $K$-spaces. The functor rig $_{\mathrm{B} 1}$ will be called the Raynaud equivalence. Composing ur' with a quasiinverse of $\operatorname{rig}_{\mathrm{Bl}}$, we obtain a functor ur: $\operatorname{Rig}_{K}^{\prime} \rightarrow \mathrm{uRig}_{K}$; if $Y$ is in $\operatorname{Rig}_{K}^{\prime}$, we say that $Y^{\mathrm{ur}}:=\operatorname{ur}(Y)$ is the uniformly rigid $K$-space associated to $Y$. Of course, it depends on the choice of a quasiinverse of the Raynaud equivalence.

Proposition 2.59. The composite functor $\mathrm{r} \circ$ ur is quasiisomorphic to the identity on $\operatorname{Rig}_{K}^{\prime}$. 
Proof. Let rig $_{\mathrm{Bl}}^{-1}$ denote the chosen inverse of the Raynaud equivalence. Let $Y$ be an object of $\operatorname{Rig}_{K}^{\prime}$; then $\operatorname{rig}_{\mathrm{Bl}}^{-1}(Y)$ is a quasiparacompact flat formal $R$-model of locally tf type for $Y$, and $Y^{\mathrm{ur}}=\operatorname{rig}_{\mathrm{Bl}}^{-1}(Y)^{\mathrm{urig}}$, which implies that $\left(Y^{\mathrm{ur}}\right)^{\mathrm{r}}=\operatorname{rig}_{\mathrm{Bl}}^{-1}(Y)^{\mathrm{rig}}$, functorially in $Y$. That is, $\mathrm{r} \circ \mathrm{ur}=\operatorname{rig} \circ \operatorname{rig}_{\mathrm{Bl}}^{-1}$, which is isomorphic to the identity functor.

In particular, after choosing an isomorphism rour $\cong \mathrm{id}$, the comparison morphisms comp $\mathrm{Yur}_{\mathrm{ur}}$ induce functorial comparison morphisms

$$
\operatorname{comp}_{Y}: Y \cong\left(Y^{\mathrm{ur}}\right)^{r} \rightarrow Y^{\mathrm{ur}}
$$

for all quasiparacompact and quasiseparated $\operatorname{rigid} K$-spaces $Y$.

Corollary 2.60. For $Y \in \operatorname{Rig}_{K}^{\prime}$, the morphism comp is $_{Y}$ the initial morphism from $Y$ to a uniformly rigid $K$-space.

Proof. Let $X$ be a uniformly rigid $K$-space, and let $\psi: Y \rightarrow X$ be a morphism

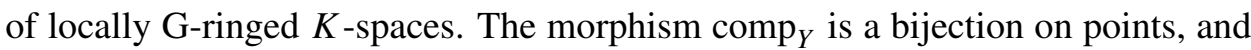
it induces isomorphisms of stalks; hence the morphism $Y^{\mathrm{ur}} \rightarrow X$ that we seek is unique if it exists. If $Y$ is affinoid and $X$ is semiaffinoid, there is nothing to show. Let $\left(X_{i}\right)_{i \in I}$ be an admissible semiaffinoid covering of $X$, and let $\left(Y_{j}\right)_{j \in J}$ be an admissible affinoid covering of $Y$ refining $\left(\psi^{-1}\left(X_{i}\right)\right)_{i \in I}$. It suffices to see that $\left(Y_{j}^{\mathrm{ur}}\right)_{j \in J}$ is an admissible covering of $Y^{\mathrm{ur}}$. By [Bosch 2005, Lemma 2.8/4], there exists a flat quasiparacompact $R$-model of locally tf type $\mathfrak{Y}$ for $Y$ such that $\left(Y_{j}\right)_{j \in J}$ is induced by an open covering of $\mathfrak{Y}$. Since $\mathfrak{Y}^{\text {urig }}=Y^{\text {ur }}$, it follows that $\left(Y_{j}^{\text {ur }}\right)_{j \in J}$ is an admissible covering of $Y^{\mathrm{ur}}$, as desired.

Corollary 2.61. The functor ur is fully faithful.

Proof. Let $X$ and $Y$ be objects in $\operatorname{Rig}_{K}^{\prime}$. By Proposition 2.59, by the global variant of Proposition 2.55 and by Corollary 2.60, we have functorial bijections

$$
\operatorname{Hom}(Y, X) \cong \operatorname{Hom}\left(Y,\left(X^{\mathrm{ur}}\right)^{\mathrm{r}}\right) \cong \operatorname{Hom}\left(Y, X^{\mathrm{ur}}\right) \cong \operatorname{Hom}\left(Y^{\mathrm{ur}}, X^{\mathrm{ur}}\right) .
$$

Of course, if $X$ is any uniformly rigid $K$-space, then the comparison morphism

$$
\operatorname{comp}_{X}: X^{\mathrm{r}} \rightarrow X
$$

is not initial all morphisms from $X^{\mathrm{r}}$ to uniformly rigid $K$-spaces. For example, if $X$ is the semiaffinoid open unit disc $\operatorname{sSp}\left(R \llbracket S \rrbracket \otimes_{R} K\right)$, then the natural morphism comp $_{X^{\mathrm{r}}}$ from the rigid open unit disc $X^{\mathrm{r}}$ to its uniform rigidification $\left(X^{\mathrm{r}}\right)^{\mathrm{ur}}$ does not extend to a morphism $X \rightarrow\left(X^{\mathrm{r}}\right)^{\mathrm{ur}}$. Indeed, such a morphism would have to be the identity on points, but $X$ is quasicompact, while $\left(X^{\mathrm{r}}\right)^{\mathrm{ur}}$ is not quasicompact.

The functor $Y \mapsto Y^{\text {ur }}$ does not respect arbitrary open immersions. For example, if $Y^{\prime} \subseteq Y$ is the inclusion of the open rigid unit disc into the closed rigid unit disc, the morphism $\left(Y^{\prime}\right)^{\mathrm{ur}} \rightarrow Y^{\mathrm{ur}}$ is not an open immersion: its image is the semiaffinoid 
open unit disc, while $\left(Y^{\prime}\right)^{\text {ur }}$ is not quasicompact. However, it follows from [Bosch and Lütkebohmert 1993b, 5.7] that ur preserves open immersions of quasicompact rigid $K$-spaces.

Quasiseparated rigid $K$-spaces are obtained from affinoid $K$-spaces by gluing along quasicompact admissible open subspaces, it thus follows that ur preserves fibered products. Indeed, this can now be checked in an affinoid situation, where the statement is clear from the construction of semiaffinoid fibered products. In particular, $Y \mapsto Y^{\mathrm{ur}}$ preserves group structures.

\section{Coherent modules on uniformly rigid spaces}

Let $X$ be a G-ringed $K$-space, and let $\mathscr{F}_{F}$ be an $\mathscr{O}_{X}$-module. Let us recall some standard definitions concerning the coherence property [Bosch 2005, 1.14/2] :

(i) $\mathscr{F}$ is called of finite type if there exists an admissible covering $\left(X_{i}\right)_{i \in I}$ of $X$ together with exact sequences

$$
\left.\mathrm{O}_{X}^{s_{i}}\right|_{X_{i}} \rightarrow \mathscr{F}_{X_{i}} \rightarrow 0 \text {. }
$$

(ii) $\mathscr{F}$ is called coherent if $\mathscr{F}$ is of finite type and if for any admissible open subspace $U \subseteq X$, the kernel of any morphism $\left.\left.O_{X}^{S}\right|_{U} \rightarrow \mathscr{F}\right|_{U}$ is of finite type.

If $X$ is a semiaffinoid $K$-space with ring of functions $A$, then the functor $M \mapsto \tilde{M}$ on the category of finite $A$-modules is well-behaved, as it is shown by the following lemma. The proof of Lemma 3.1 is identical to the proof of [Bosch 2005, 1.14/1]; one uses the fact that the restriction homomorphisms associated to semiaffinoid subdomains are flat:

Lemma 3.1. The functor $M \mapsto \tilde{M}$ from the category of finite A-modules to the category of $\mathrm{O}_{X}$-modules is fully faithful, and it commutes with the formation of kernels, images, cokernels and tensor products. Moreover, a sequence of finite A-modules

$$
0 \rightarrow M^{\prime} \rightarrow M \rightarrow M^{\prime \prime} \rightarrow 0
$$

is exact if and only if the associated sequence

$$
0 \rightarrow \tilde{M}^{\prime} \rightarrow \tilde{M} \rightarrow \tilde{M}^{\prime \prime} \rightarrow 0
$$

of $\mathrm{O}_{X}$-modules is exact.

For a semiaffinoid $K$-space $X=\operatorname{sSp} A$, we have $\mathscr{O}_{X}^{r}=A^{r} \otimes \mathcal{O}_{X}$. Since $A$ is noetherian, it follows from Lemma 3.1 that kernels and cokernels of morphisms of type $\mathbb{O}_{X}^{r} \rightarrow \mathcal{O}_{X}^{s}$ are associated. We thus conclude that an $\mathbb{O}_{X}$-module on a uniformly rigid $K$-space $X$ is coherent if and only if there exists an admissible semiaffinoid covering $\left(X_{i}\right)_{i \in I}$ of $X$ such that $\left.\mathscr{F}\right|_{X_{i}}$ is associated for all $i \in I$. 
In particular, the structural sheaf $0_{X}$ of any uniformly rigid $K$-space $X$ is coherent. Moreover, it follows from Lemma 3.1 that kernels and cokernels of morphisms of coherent $\mathbb{O}_{X}$-modules are coherent.

Lemma 3.2. Let $\varphi: Y \rightarrow X$ be a morphism of uniformly rigid $K$-spaces, and let $\mathscr{F}$ be a coherent $\mathrm{O}_{X}$-module. Then $\varphi^{*} \mathscr{F}$ is a coherent $\mathrm{O}_{Y}$-module.

Proof. Indeed, we may assume that $X$ and $Y$ are semiaffinoid, with $X=\operatorname{sSp} A$ and $Y=\operatorname{sSp} B$, and that $\mathscr{F}_{F}$ is associated to a finite $A$-module $M$. Then $\varphi^{*} \mathscr{F}_{F}$ is associated to $M \otimes_{A} B$, where $B$ is an $A$-algebra via $\varphi^{*}$.

Definition 3.3. Let $X$ be a uniformly rigid $K$-space. An $\mathscr{O}_{X}$-module $\mathscr{F}$ is called strictly coherent if for any open semiaffinoid subspace $U \subseteq X$, the restriction $\left.\mathscr{F}_{\mid}\right|_{U}$ is an associated module.

For example, the structural sheaf of a uniformly rigid $K$-space is strictly coherent. Since we do not know whether an open semiaffinoid subspace of a semiaffinoid $K$-space is a semiaffinoid subdomain, it is not a priori clear whether any associated module on a semiaffinoid $K$-space is strictly coherent. In Corollary 3.6, however, we will show that this is indeed the case.

Let $X$ be a uniformly rigid $K$-space. We will be interested in coherent $\mathrm{O}_{X}$ modules $\mathscr{F}_{F}$ with the property that there exists an injective $\mathcal{O}_{X}$-homomorphism $\mathscr{F}_{\hookrightarrow} \hookrightarrow$ $\mathcal{O}_{X}^{r}$ for some $r \in \mathbb{N}$. This property is clearly satisfied by coherent ideals, and it is preserved under pullback with respect to flat morphisms of uniformly rigid spaces. We will study integral models of such $\mathscr{F}$, and we will show that any such $\mathscr{F}$ is strictly coherent.

If $\mathfrak{X}$ is a formal $R$-scheme of locally ff type and if $\underline{\mathscr{F}}$ is a coherent $O_{\mathfrak{X}}$-module, we obtain a coherent $O_{X}$-module $\underline{F}^{\text {urig }}$ on $\mathfrak{X}^{\text {urig }}$ which we call the uniformly rigid generic fiber of $\underline{\mathscr{F}}$. If $X$ is a uniformly rigid $K$-space, if $\mathscr{F}$ is a coherent $\mathcal{O}_{X}$-module and if $\mathfrak{X}$ is a flat formal $R$-model of locally ff type for $X$, then an $R$-model of $\mathscr{F}$ on $\mathfrak{X}$ is a coherent $O_{\mathfrak{X}}$-module $\mathscr{F}$ together with an isomorphism $\mathscr{F}^{\text {urig }} \cong \mathscr{F}$ that is compatible with the given identification $\mathfrak{X}^{\text {urig }} \cong X$. Sometimes we will not mention the isomorphism $\underline{\mathscr{F}}^{u r i g} \cong \mathscr{F}$ explicitly. Clearly

$$
\operatorname{sp}_{\mathfrak{X}, *}(\mathscr{F})=\underline{\mathscr{F}} \otimes_{R} K,
$$

and urig factors naturally through the functor $\underline{\mathscr{F}} \mapsto \underline{\mathscr{F}} \otimes_{R} K$. Let us abbreviate $\underline{\mathscr{F}}_{K}:=\underline{\mathscr{F}}_{{ }_{R}} K$.

For any $r \in \mathbb{N}$, the coherent $\mathcal{O}_{X}$-module $\mathscr{O}_{X}^{r}$ admits the natural model $\mathscr{O}_{\mathfrak{X}}^{r}$ on every flat formal $R$-model of locally ff type $\mathfrak{X}$ for $X$. We will show that coherent submodules $\mathscr{F} \subseteq \mathrm{O}_{X}^{r}$ inherit this property by taking schematic closures. Let us first consider the affine situation:

Lemma 3.4. Let $\underline{A}$ be an $R$-algebra, let $\underline{M}$ be an $\underline{A}$-module, and let $N \subseteq \underline{M} \otimes_{R} K$ be an $\underline{A} \otimes_{R} K$-submodule. Then there exists a unique $\underline{A}$-submodule $\underline{N} \subseteq \underline{M}$ such 
that the natural homomorphism $\underline{N} \otimes_{R} K \rightarrow \underline{M} \otimes_{R} K$ is an isomorphism onto $N$ and such that $\underline{M} / \underline{N}$ is $R$-flat.

Proof. Let us abbreviate $M:=\underline{M} \otimes_{R} K$, and let us set

$$
\underline{N}:=\operatorname{ker}(\underline{M} \rightarrow M / N)
$$

then $\underline{N}$ is an $\underline{A}$-submodule of $\underline{M}$. For any $n \in N$, there exists an $s \in \mathbb{N}$ such that $\pi^{s} n$ lies in the image of $\underline{M}$ in $M$; the natural $K$-homomorphism $\underline{N} \otimes_{R} K \rightarrow N$ is thus bijective. As an $\underline{A}$-submodule of $M / N$, the quotient $\underline{M} / \underline{N}$ is free of $\pi$-torsion and, hence, $R$-flat.

If $\underline{N}^{\prime} \subseteq \underline{M}$ is another $\underline{A}$-submodule whose image in $M$ generates $N$ as an $\underline{A} \otimes_{R} K$ module, then $\underline{N}^{\prime}$ lies in the kernel $\underline{N}$ of $\underline{M} \rightarrow M / N$. If in addition $\underline{M} / \underline{N}^{\prime}$ is flat over $R$, then the natural homomorphism $\underline{M} / \underline{N}^{\prime} \rightarrow \underline{M} / \underline{N}^{\prime} \otimes_{R} K=M / N$ is injective, which proves that $\underline{N}^{\prime}$ coincides with this kernel.

Theorem 3.5. Let $X$ be a uniformly rigid $K$-space, let $\mathscr{F}^{\prime} \subseteq \mathscr{F}$ be an inclusion of coherent $\mathrm{O}_{X}$-modules, and let $\mathfrak{X}$ be an $R$-model of locally ff type for $X$ such that $\mathscr{F}_{F}$ admits be an $R$-model $\mathscr{F}$ on $\mathfrak{X}$. Then there exists a unique coherent $\mathcal{O}_{\mathfrak{X}}$-submodule $\underline{\mathscr{F}}^{\prime} \subseteq \underline{\mathscr{F}}$ such that $\underline{\mathscr{F}} / \underline{\mathscr{F}}^{\prime}$ is $R$-flat and such that the given isomorphism $\underline{\mathscr{F}}^{\mathrm{urig}} \cong \mathscr{F}$ identifies $\left(\underline{\mathscr{F}}^{\prime}\right)^{\text {urig }}$ with $\mathscr{F}^{\prime}$.

Proof. We may work locally on $\mathfrak{X}$ and thereby assume that $\mathfrak{X}$ is affine. Uniqueness of $\mathscr{F}^{\prime}$ is a consequence of Lemma 3.4. Since $\mathscr{F}^{\prime}$ is coherent, there exists a treelike covering $\left(X_{i}\right)_{i \in I}$ of $X$ such that $\left.\mathscr{F}^{\prime}\right|_{X_{i}}$ is associated for all $i \in \operatorname{lv}(I)$. Let us choose a model of this covering, that is,

(i) for each $i \in I$, an affine flat $R$-model of ff type $\mathfrak{X}_{i}$ for $X_{i}$,

(ii) for each inner $i \in I$ an admissible blowup $\beta_{i}: \mathfrak{X}_{i}^{\prime} \rightarrow \mathfrak{X}_{i}$ and

(iii) for each inner $i \in I$ and for each child $j$ of $i$ an open immersion $\varphi_{j}: \mathfrak{X}_{j} \hookrightarrow \mathfrak{X}_{i}^{\prime}$ such that $\mathfrak{X}_{j} \subseteq \mathfrak{X}_{i}^{\prime} \rightarrow \mathfrak{X}_{i}$ represents $X_{j}$ in $X_{i}$.

For each $i \in I$, we let $\left.\underline{\mathscr{F}}\right|_{\mathfrak{X}_{i}}$ denote the pullback of $\underline{\mathscr{F}}_{\mathrm{F}}$ to $\mathfrak{X}_{i}$, and for each inner vertex $i \in I$, we let $\left.\underline{\mathscr{F}}\right|_{\mathfrak{X}_{i}^{\prime}}$ denote the pullback of $\mathscr{F}$ to $\mathfrak{X}_{i}^{\prime}$. Let $i$ be an inner vertex of $I$, and let us assume that for each child $j$ of $i$, we are given a coherent submodule

$$
\underline{\mathscr{F}}_{j}^{\prime} \subseteq \underline{\mathscr{F}}_{\mathfrak{X}_{j}}
$$

such that $\underline{\mathscr{F}}_{\mathfrak{X}_{j}} / \underline{\mathscr{F}}_{j}^{\prime}$ is $R$-flat and such that $\left(\underline{\mathscr{F}}_{j}^{\prime}\right)^{\text {urig }}=\left.\mathscr{F}^{\prime}\right|_{X_{j}}$. By Lemma 3.4, this assumption is satisfied if all children of $i$ are leaves in $I$. Using the uniqueness assertion in Lemma 3.4, we see that the $\underline{\mathscr{F}}_{j}^{\prime}$ glue to a unique coherent submodule

$$
\left.\underline{\varphi}_{i} \subseteq \underline{\mathscr{F}^{\prime}}\right|_{\mathfrak{X}_{i}^{\prime}} .
$$

The quotient $\left.\underline{\mathscr{F}}\right|_{\mathfrak{X}_{i}^{\prime}} / \underline{\mathscr{G}}_{i}$ is $R$-flat; by [Grothendieck 1961b, 3.4.2], $\beta_{i *}\left(\left.\underline{\mathscr{F}}_{\mid}\right|_{\mathfrak{X}_{i}^{\prime}} / \underline{\mathscr{G}}_{i}\right)$ thus is a coherent $R$-flat $O_{\mathfrak{X}_{i}}$-module. By definition, $\left.\underline{\mathscr{F}}\right|_{\mathfrak{X}_{i}^{\prime}}=\left.\beta_{i}^{*} \underline{\mathscr{F}}\right|_{\mathfrak{X}_{i}}$, so we have a natural 
homomorphism of coherent $\mathrm{O}_{\mathfrak{X}_{j}}$-modules

$$
\underline{\mathscr{F}}\left|\mathfrak{X}_{i} \rightarrow \beta_{i *} \underline{\mathscr{F}}\right|_{\mathfrak{X}_{i}^{\prime}} \rightarrow \beta_{i *}\left(\left.\underline{\mathscr{F}}\right|_{\mathfrak{X}_{i}^{\prime}} / \underline{\mathscr{G}}_{i}\right) \text {. }
$$

Let $\underline{\mathscr{F}}_{i}^{\prime}$ denote its kernel; the resulting exact sequence of coherent $\mathcal{O}_{\mathfrak{X}_{i}}$-modules

$$
0 \rightarrow \underline{\mathscr{F}}_{i}^{\prime} \rightarrow \underline{\mathscr{F}} \mid \mathfrak{X}_{i} \rightarrow \beta_{i *}\left(\left.\underline{\mathscr{F}}_{\mid}\right|_{\mathfrak{X}_{i}^{\prime}} / \underline{G}_{i}\right)
$$

shows that $\underline{\mathscr{F}}_{\mid} \mathfrak{X}_{i} / \underline{\mathscr{F}}_{i}^{\prime}$ is $R$-flat. We claim that the coherent $X_{i}$-module $\left.\mathscr{F}^{\prime}\right|_{X_{i}}=\underline{\varphi}_{i}^{\text {urig }}$ is associated to $\underline{\mathscr{F}}_{i}^{\prime}$. To prove this, it suffices to show that the morphism

$$
\left(\beta_{i}^{*} \underline{\mathscr{F}}_{i}^{\prime}\right)_{K} \rightarrow\left(\beta_{i}^{*} \beta_{i *} \underline{\varphi}_{i}\right)_{K} \rightarrow \underline{\varphi}_{i, K}
$$

induced by the natural morphism $\underline{\mathscr{F}}_{i}^{\prime} \rightarrow \beta_{i *} \underline{\underline{G}}_{i}$ is an isomorphism. By the ff type variant of [Lütkebohmert 1990, 2.1], the second morphism in (\$) is an isomorphism, so we must show that the first morphism is an isomorphism as well. Let $\underline{X}_{i}$ be the spectrum of the ring of global functions on $\mathfrak{X}_{i}$, and let $b_{i}: \underline{X}_{i}^{\prime} \rightarrow \underline{X}_{i}$ be the admissible blowup such that $\beta_{i}=b_{i}^{\wedge}$, where we use a wedge to denote the formal completion with respect to an ideal of definition of $\mathfrak{X}$. Let $\underline{F}_{i}, \underline{F}_{i}{ }^{\prime}$ and $\underline{G}_{i}$ denote the algebraizations of $\underline{\mathscr{F}}_{\mathfrak{X}_{i}}, \underline{\mathscr{F}}_{i}^{\prime}$ and $\underline{G}_{i}$ respectively, which exist by [Grothendieck 1961b, 5.1.4]; then

$$
\left.\underline{\mathscr{F}_{F}}\right|_{\mathfrak{X}_{i}^{\prime}}=\left(b_{j}^{*} \underline{F}_{i}\right)^{\wedge} .
$$

By [Grothendieck 1961b, 4.1.5],

$$
\left.\beta_{i *}\left(\left.\underline{\mathscr{F}}_{\mid}\right|_{i} ^{\prime} / \underline{G}_{i}\right)=\left(b_{i *}\left(\left(b_{i}^{*} \underline{F}_{i}\right) / \underline{G}_{i}\right)\right)\right)^{\wedge},
$$

so we have a short exact sequence

$$
\left.0 \rightarrow \underline{F}^{\prime}{ }_{i} \rightarrow \underline{F}_{i} \rightarrow b_{i *}\left(\left(b_{i}^{*} \underline{F}_{i}\right) / \underline{G}_{i}\right)\right)
$$

which under $\cdot \otimes_{R} K$ induces a short exact sequence

$$
\left.0 \rightarrow \underline{F}^{\prime}{ }_{i, K} \rightarrow \underline{F}_{i, K} \rightarrow\left(b_{i, K}\right)_{*}\left(\left(b_{i, K}^{*} \underline{F}_{i, K}\right) / \underline{G}_{i, K}\right)\right) .
$$

Since $b_{i, K}$ is an isomorphism and, hence, flat, we obtain an induced short exact sequence

$$
\left.0 \rightarrow b_{i, K}^{*} \underline{F}_{i, K}^{\prime} \rightarrow b_{i, K}^{*} \underline{F}_{i, K} \rightarrow b_{i, K}^{*}\left(b_{i, K}\right)_{*}\left(\left(b_{i, K}^{*} \underline{F}_{i, K}\right) / \underline{G}_{i, K}\right)\right) ;
$$

since $b_{i, K}^{*}\left(b_{i, K}\right)_{*}$ is naturally isomorphic to the identity functor, this shows that $b_{i, K}^{*} \underline{F}_{i, K}^{\prime}=\underline{G}_{i, K}$. Hence, the natural morphism

$$
b_{i}^{*} \underline{F}_{j}^{\prime} \rightarrow b_{i}^{*} b_{i *} \underline{G}_{j}
$$


becomes an isomorphism under $\cdot \otimes_{R} K$. That is, its kernel and cokernel are $\pi$ torsion. It follows that kernel and cokernel of the completed morphism

$$
\beta_{i}^{*} \underline{\mathscr{F}}_{i}^{\prime} \rightarrow \beta_{i}^{*} \beta_{i * \underline{G}_{i}}
$$

are $\pi$-torsion as well, which yields our claim.

Let us now prove the statement of the proposition by induction on the volume $v(I)$ of $I$. We may assume that $I$ has more than one vertex. Let $j$ be a leaf of $I$ whose path to the root has maximal length, and let $i$ be the parent of $j$. Then all children of $i$ are leaves of $I$, so the assumption in the argument above is satisfied. By what we have shown so far, $\left.\mathscr{F}^{\prime}\right|_{X_{i}}$ is associated to a unique coherent $\mathcal{O}_{\mathfrak{X}_{i}}$-submodule $\left.\underline{\mathscr{F}}_{i}^{\prime} \subseteq \underline{\mathscr{F}^{\prime}}\right|_{\mathfrak{X}_{i}}$ such that $\underline{\mathscr{F}}_{\mathfrak{X}_{i}} / \underline{\mathscr{F}}_{i}^{\prime}$ is $R$-flat. We may thus replace subt $(i)$ by $\{i\}$. By induction on $v(I)$, the desired statement follows.

Corollary 3.6. We conclude:

(i) A coherent submodule of an associated module on a semiaffinoid $K$-space is associated.

(ii) Coherent submodules and coherent quotients of strictly coherent modules are strictly coherent.

(iii) An associated module on a semiaffinoid $K$-space is strictly coherent.

Proof. Let us first show (i). Let $X=\mathrm{sSp} A$ be a semiaffinoid $K$-space, let $\underline{A} \subseteq A$ be an $R$-model of ff type, and let $\mathscr{F}^{\prime}$ be a coherent submodule of an associated module $\tilde{M}$. Since $\tilde{M}$ admits a model $\underline{M}$ over $\operatorname{Spf} \underline{A}$, Theorem 3.5 implies that $\mathscr{F}^{\prime} \cong\left(\mathscr{F}^{\prime}\right)^{\text {urig }}$ for a coherent module $\underline{\mathscr{F}}^{\prime}$ on Spf $\underline{A}$. Since coherent modules on affine formal schemes are associated, it follows that $\mathscr{F}^{\prime}$ is associated.

Let us prove statement (ii). Let $X$ be a uniformly rigid $K$-space, let $\mathscr{F}$ be a strictly coherent $O_{X}$-module and let $\mathscr{F}^{\prime} \subseteq \mathscr{F}$ be a coherent submodule. For every open semiaffinoid subspace $U \subseteq X$, the restriction $\left.\mathscr{F}^{\prime}\right|_{U}$ is a coherent submodule of $\left.\mathscr{F}\right|_{U}$, and $\left.\mathscr{F}\right|_{U}$ is associated by assumption on $\mathscr{F}$. It follows from (i) that $\left.\mathscr{F}^{\prime}\right|_{U}$ is associated; hence $\mathscr{F}^{\prime}$ is strictly coherent. Let now $\mathscr{F}^{\prime \prime}$ be a coherent quotient of $\mathscr{F}$. Then the kernel $\mathscr{F}^{\prime}$ of the projection $\mathscr{F}^{\rightarrow} \mathscr{F}^{\prime \prime}$ is a coherent submodule of $\mathscr{F}^{\prime}$ and, hence, strictly coherent by what we have seen so far. Let $U \subseteq X$ be an open semiaffinoid subspace; then we have a short exact sequence

$$
\left.\left.\left.0 \rightarrow \mathscr{F}^{\prime}\right|_{U} \rightarrow \mathscr{F}\right|_{U} \rightarrow \mathscr{F}^{\prime \prime}\right|_{U} \rightarrow 0
$$

where the first two modules are associated. It follows from Lemma 3.1 that $\left.\mathscr{F}^{\prime \prime}\right|_{U}$ is associated as well.

Finally, statement (iii) follows from statement (ii) because by Lemma 3.1, an associated module is a quotient of a finite power of the structural sheaf.

If $\mathfrak{X}$ is a flat formal $R$-scheme of locally ff type and if $\mathscr{F}$ is a coherent $\mathfrak{O}_{\mathfrak{X}}$-module, we do not know in general whether $\underline{\mathscr{F}}^{\text {urig }}$ is strictly coherent. In particular, we 
unfortunately do not know whether the analog of Kiehl's theorem [Kiehl 1967, 1.2] holds in general, that is to say whether every coherent module on a semiaffinoid $K$ space is associated. Let us point out that the analogous question for quasicoherent modules on rigid spaces was open for a long time; it was finally settled in the negative by O. Gabber [Conrad 2006, Example 2.1.6].

Conjecture 3.7. The general uniformly rigid analog of Kiehl's theorem does not hold.

Remark 3.8. The general uniformly rigid analog of Kiehl's theorem is equivalent to the following statement: let $\mathfrak{X}$ be an admissible blowup of a flat affine formal $R$-scheme of ff type, and let $\mathscr{F}$ be a coherent sheaf on $X=\mathfrak{X}^{\text {urig }}$ that admits flat models $\underline{\mathscr{F}}_{i}$ locally with respect to an affine open covering $\left(\mathfrak{X}_{i}\right)_{i \in I}$ of $\mathfrak{X}$; then $\mathscr{F}_{F}$ admits a model on $\mathfrak{X}$. Indeed, this equivalence follows by arguing as in the proof of [Lütkebohmert 1990, Theorem 2.3]. However, it seems impossible in general to modify the models $\underline{\mathscr{F}}_{i}$ such that they glue to a model of $\mathscr{F}$ on $\mathfrak{X}$ : Let us assume that $I=\{1,2\}$. After multiplying $\mathscr{\mathscr { F }}_{1}$ by a suitable power of $\pi$, we may assume that $\underline{\mathscr{F}}_{1}$ is contained in $\underline{\mathscr{F}}_{2}$ on the intersection $\mathfrak{X}_{12}$ of $\mathfrak{X}_{1}$ and $\mathfrak{X}_{2}$. Let $n \in \mathbb{N}$ be big enough such that $\pi^{n} \underline{\mathscr{F}}_{2} \subseteq \underline{\mathscr{F}}_{1}$ on $\mathfrak{X}_{12}$; then $\underline{\mathscr{G}}:=\underline{\mathscr{F}}_{1}\left|\mathfrak{X}_{12} / \pi^{n} \underline{\mathscr{F}}_{2}\right|_{\mathfrak{X}_{12}}$ is a coherent subsheaf of $\left.\left(\underline{\mathscr{F}}_{2} / \pi^{n} \underline{\mathscr{F}}_{2}\right)\right|_{\mathfrak{X}_{12}}$; see the proof of [Lütkebohmert 1990, Lemma 2.2]. If $\mathfrak{X}$ is of tf type over $R$, then the closed formal subscheme of $\mathfrak{X}$ cut out by $\pi^{n}$ is a scheme, and by chasing denominators [Grothendieck and Dieudonné 1960, 9.4.7] one can extend $\underline{G}$ to a coherent subsheaf, again denoted by $\underline{\mathscr{G}}$, on all of $\mathfrak{X}_{2}$. Let $\underline{\mathscr{F}}_{2}^{\prime}$ denote the preimage of $\underline{G}_{1}$ under the projection $\underline{\mathscr{F}}_{2} \rightarrow \underline{\mathscr{F}}_{2} / \pi^{n} \underline{\mathscr{F}}_{2}$; then $\underline{\mathscr{F}}_{2}^{\prime}$ is a model of $\mathscr{F}_{F}$ on $\mathfrak{X}_{2}$ which glues to $\underline{\mathscr{F}}_{1}$, and we obtain a model of $\mathscr{F}_{F}$ on all of $\mathfrak{X}$. In our situation, however, $\mathfrak{X}_{2}$ might not be of tf type, and hence the closed formal subscheme of $\mathfrak{X}_{2}$ cut out by $\pi^{n}$ might not be a scheme. On a formal scheme though it is in general not possible to extend coherent subsheaves because of convergence problems. Thus, Lütkebohmert's proof of Kiehl's theorem fails in the uniformly rigid situation. Similar problems occur if one tries to carry over Kiehl's original proof.

\section{A. Closed uniformly rigid subspaces.}

Definition 3.9. A morphism of uniformly rigid $K$-spaces $\varphi: Y \rightarrow X$ is called a closed immersion if there exists an admissible semiaffinoid covering $\left(X_{i}\right)_{i \in I}$ of $X$ such that for each $i \in I$, the restriction $\varphi^{-1}\left(X_{i}\right) \rightarrow X_{i}$ of $\varphi$ is a closed immersion of semiaffinoid $K$-spaces in the sense of Definition 2.19.

We easily see that closed immersions are injective on the level of physical points.

Lemma 3.10. Let $\varphi: Y \rightarrow X$ be a closed immersion of uniformly rigid $K$-spaces. Then $\varphi^{\sharp}: O_{X} \rightarrow \varphi_{*} O_{Y}$ is an epimorphism of sheaves. Moreover, the $O_{X}$-modules $\varphi_{*} \mathrm{O}_{Y}$ and $\operatorname{ker} \varphi^{\sharp}$ are strictly coherent. 
Proof. The $\mathrm{O}_{X}$-module $\mathrm{O}_{X}$ is strictly coherent. By Corollary 3.6(ii), it thus suffices to show that $\varphi^{\sharp}$ is an epimorphism and that both $\operatorname{ker} \varphi^{\sharp}$ and $\varphi_{*} O_{Y}$ are coherent. Considering an admissible semiaffinoid covering $\left(X_{i}\right)_{i \in I}$ of $X$ such that for all $i \in I$, the restriction $\varphi^{-1}\left(X_{i}\right) \rightarrow X_{i}$ of $\varphi$ is a closed immersion of semiaffinoid $K$-spaces, we reduce to the case where both $X$ and $Y$ are semiaffinoid and where $\varphi$ corresponds to a surjective homomorphism of semiaffinoid $K$-algebras. Now the desired statements follow from Lemma 3.1.

Proposition 3.11. Let $\varphi: Y \rightarrow X$ be a morphism of uniformly rigid $K$-spaces. Then the following are equivalent:

(i) $\varphi$ is a closed immersion.

(ii) For each open semiaffinoid subspace $U \subseteq X$, the restriction $\varphi^{-1}(U) \rightarrow U$ is a closed immersion of semiaffinoid $K$-spaces in the sense of Definition 2.19.

Proof. The implication (ii) $\Rightarrow$ (i) is trivial, the open semiaffinoid subspaces forming a basis for the G-topology on $X$. Let us assume that (i) holds, let $\mathscr{I}$ denote the kernel of $\varphi^{\sharp}$, and let $U \subseteq X$ be an open semiaffinoid subspace; then $\varphi$ induces a short exact sequence

$$
\left.\left.0 \rightarrow \mathscr{I}\right|_{U} \rightarrow \mathrm{O}_{U} \rightarrow \varphi_{*} \mathrm{O}_{Y}\right|_{U} \rightarrow 0 .
$$

Let $A$ denote the ring of functions on $U$. By Lemma 3.10, $\mathscr{I}$ and $\varphi_{*} \mathcal{O}_{Y}$ are strictly coherent; hence the above short exact sequence is associated to a short exact sequence of $A$-modules

$$
0 \rightarrow I \rightarrow A \rightarrow B \rightarrow 0 .
$$

Since morphisms from uniformly rigid $K$-spaces to semiaffinoid $K$-spaces correspond to $K$-homomorphisms of rings of global functions, we can now mimic the proof of [Bosch et al. 1984, 9.4.4/1] to see that the restriction $\varphi^{-1}(U) \rightarrow U$ of $\varphi$ is associated to the projection $A \rightarrow B$ : it suffices to see that the natural morphism $\varphi^{-1}(U) \rightarrow \operatorname{sSp} B$ is an isomorphism. This can be checked locally on $\operatorname{sSp} B$ with respect to the preimage under $\operatorname{sSp} B \rightarrow U$ of a leaflike refinement of $\left(U \cap X_{i}\right)_{i \in I}$, where $\left(X_{i}\right)_{i \in I}$ is an admissible semiaffinoid covering of $X$ satisfying the conditions of Definition 3.9.

Remark 3.12. The proof of [Bosch et al. 1984, 9.4.4/1] resorts to [ibid., 8.2.1/4]. However, as our argument above shows, this is in fact unnecessary - which is to our advantage, because the statement of 8.2.1/4 fails to hold in the semiaffinoid situation: Example 2.42 yields a bijective morphism of semiaffinoid $K$-spaces which induces isomorphisms of stalks and which is not an isomorphism.

In particular, a morphism of semiaffinoid $K$-spaces is a closed immersion in the sense of Definition 3.9 if and only if it is a closed immersion of semiaffinoid $K$-spaces in the sense of Definition 2.19. We can now define a closed uniformly 
rigid subspace as an equivalence class of closed immersions, in the usual way. By standard gluing arguments, we see that the closed uniformly rigid subspaces of a uniformly rigid $K$-space $X$ correspond to the coherent $0_{X}$-ideals. We easily see that closed immersions of uniformly rigid $K$-spaces are preserved under base change.

It is clear that closed immersions of formal $R$-schemes of locally ff type induce closed immersions on uniformly rigid generic fibers. Conversely, given a uniformly rigid $K$-space $X$ together with an $R$-model of locally ff type $\mathfrak{X}$ and a closed uniformly rigid subspace $V \subseteq X$, there exists a unique $R$-flat closed formal subscheme $\mathfrak{V} \subseteq \mathfrak{X}$ such that the given isomorphism $\mathfrak{X}^{\text {urig }} \cong X$ identifies $\mathfrak{V}^{\text {urig }}$ with $V$. Indeed, this is an immediate consequence of Theorem 3.5. We say that $\mathfrak{V}$ is the schematic closure of $V$ in $\mathfrak{X}$.

The comparison functors studied in Section 2D1 preserve closed immersions. This can be verified in the semiaffinoid and affinoid situations respectively. In the case of the functor ur, there is nothing to show. In the case of the functor $\mathrm{r}$, the statement follows by looking at schematic closures and using the fact that Berthelot's construction preserves closed immersions [de Jong 1995, 7.2.4(e)].

3A1. Separated uniformly rigid spaces. As usual, a morphism $\varphi: Y \rightarrow X$ of uniformly rigid $K$-spaces is called separated if its diagonal morphism

$$
\Delta_{\varphi}: Y \rightarrow Y \times{ }_{X} Y
$$

is a closed immersion. A uniformly rigid $K$-space $X$ is called separated if its structural morphism $X \rightarrow \operatorname{sSp} K$ is separated. If $X$ is a uniformly rigid $K$-space, we let $\Delta_{X}$ denote the diagonal of its structural morphism.

Semiaffinoid $K$-spaces are visibly separated. Moreover, uniformly rigid generic fibers of separated morphisms of formal $R$-schemes of locally ff type are separated, since functor urig preserves fibered products and closed immersions. Similarly, the comparison functors studied in Section 2D1 preserve the separatedness property.

Lemma 3.13. Let $X$ be a separated uniformly rigid $K$-space. The intersection of two open semiaffinoid subspaces in $X$ is an open semiaffinoid subspace in $X$.

Proof. Let $U$ and $V$ be open semiaffinoid subspaces in $X$. We easily see, using points with values in finite field extensions of $K$, that $U \cap V$ is the $\Delta_{X}$-preimage of $U \times_{\operatorname{sSp} K} V$ which is an open semiaffinoid subspace of $X \times_{\operatorname{sSp} K} X$. Since $\Delta_{X}$ is a closed immersion by assumption on $X$, it follows from Proposition 3.11 that $U \cap V$ is an open semiaffinoid subspace of $X$.

Corollary 3.14. Let $X$ be a separated uniformly rigid $K$-space, and let $\mathscr{F}$ be a coherent $\mathbb{O}_{X}$-module. Then the natural morphism

$$
\check{H}^{q}(X, \mathscr{F}) \stackrel{\sim}{\rightarrow} H^{q}(X, \mathscr{F})
$$

is an isomorphism for all $q \geq 0$. 
Proof. Let $S$ denote the set of open semiaffinoid subspaces $U$ in $X$ with the property that $\mathscr{F}_{U}$ is associated. By Lemma 3.13, this set is stable under the formation of intersections. It is clearly a basis for the G-topology on $X$, and $\check{H}^{q}(U, \mathscr{F})=0$ for any $U$ in $S$ and any $q \geq 0$ by Corollary 2.43 . We conclude by the usual Čech spectral sequence argument.

If $X$ is a separated uniformly rigid $K$-space and if $\varphi: Y \rightarrow X$ is a morphism of uniformly rigid $K$-spaces, then the graph $\Gamma_{\varphi}: Y \rightarrow Y \times X$ of $\varphi$ is a closed immersion since it is obtained from $\Delta_{X}$ via pullback. In particular, if $\mathfrak{X}$ and $\mathfrak{Y}$ are $R$-models of locally ff type for $X$ and $Y$ respectively, the schematic closure of $\Gamma_{\varphi}$ in $\mathfrak{Y} \times \mathfrak{X}$ is well-defined. Here fibered products without indication of the base are understood over sSp $K$ or $\operatorname{Spf} R$ respectively.

\section{Comparison with the theories of Berkovich and Huber}

The category of formal $R$-schemes of locally ff type is a full subcategory of Huber's category of adic spaces [Huber 1996]. If $\mathfrak{X}$ is a formal $R$-scheme of locally $\mathrm{ff}$ type, viewed as an adic space, then by [Huber 1996, 1.2.2] the fibered product $\mathfrak{X} \times \operatorname{Spa}(R, R) \operatorname{Spa}(K, R)$ is the adic space associated to the rigid generic fiber $\mathfrak{X}^{\text {rig }}$ of $\mathfrak{X}$. That is, the uniform structure induced by $\mathfrak{X}$ is lost. In fact, we do not see a way to view the category of uniformly rigid spaces as a full subcategory of Huber's category of adic spaces. The main obstacle lies in the fact that if $\underline{A}$ is an $R$-algebra of ff type, equipped with its natural Jacobson-adic topology, and if $A=\underline{A} \otimes_{R} K$, then the pair $(A, \underline{A})$ is in general not an f-adic ring in the sense of [Huber 1996]. For example, for $\underline{A}=R \llbracket S \rrbracket$ there exists no ring topology on $A$ such that $\underline{A}$ is open in this topology: There is a unique such group topology, but multiplication by $\pi^{-1}$ in $A$ is not continuous because there is no $n \in \mathbb{N}$ such that $\pi^{-1} S^{n} \in R \llbracket S \rrbracket \otimes_{R} K$ is contained in $R \llbracket S \rrbracket$.

The situation is different if we consider the $\pi$-adic topology on $R$-algebras of ff type. If $\underline{A}^{\pi}$ denotes the ring $\underline{A}$ equipped with its $\pi$-adic topology, then the pair $\left(A, \underline{A}^{\pi}\right)$ is an f-adic ring in the sense of Huber. The induced topology on $A$ is in fact a $K$-Banach algebra topology; if, for $f \in A$ nonzero, we set $v_{\underline{A}}(F):=\max \left\{n \in \mathbb{N} ; \pi^{-n} f \in \underline{A}\right\}$, then $|f|_{\underline{A}}:=|\pi|^{v_{\underline{A}}(f)}$ defines a $K$-Banach algebra norm on $A$ which induces the topology defined by $\underline{A}^{\pi}$. If $\underline{A}=R \llbracket S \rrbracket\langle T\rangle$ is a mixed formal power series ring in finitely many variables, then $|\cdot|_{R \llbracket S \|\langle T\rangle}$ is the Gauss norm, and it coincides with the supremum seminorm taken over all points in Max $A$. Using [Bosch et al. 1984, 3.7.5/2], one proves that all $K$-Banach algebra structures on $A$ are equivalent; in particular, the valuation spectrum $M(A)$ in the sense of [Berkovich 1990,1.2] is well defined. One shows that reduced semiaffinoid $K$-algebras are Banach function algebras, and one verifies that the supremum seminorm, taken over all points in Max $A$ or, equivalently, over all 
points in $M(A)$, takes values in $\sqrt{|K|}$. For a more detailed discussion, including proofs, we refer to [Kappen 2009, Section 1.2.5].

The topological space $M(A)$ may be viewed as a compactification of the rigid space $(\operatorname{sSp} A)^{\mathrm{r}}$. To illustrate this idea in terms of an example, let us first explain how the specialization map extends to valuation spectra. If $A$ is a semiaffinoid $K$ algebra and if $\underline{A}$ is an $R$-model of ff type for $A$, there exists a natural specialization map

$$
\mathrm{sp}_{\underline{A}}: M(A) \rightarrow \operatorname{Spec}(\underline{A} / \pi \underline{A})
$$

extending the specialization map which we discussed in Section 2B1: let $x$ be a point in $M(A)$, represented by a character $\chi_{x}: A \rightarrow \mathscr{K}$ with values in some valued field extension $\mathscr{K}$ of $K$; then $\operatorname{sp}_{\underline{A}}(x):=\operatorname{ker}\left(\tilde{\chi}_{x}: \underline{A} / \pi \underline{A} \rightarrow \tilde{\mathscr{K}}\right)$, where $\tilde{\mathscr{K}}$ is the residue field of $\mathscr{K}$ and where $\tilde{\chi}_{x}$ is the reduction of $\chi_{x}$.

Lemma 4.1. The map $\operatorname{sp}_{A}$ is surjective onto $\operatorname{Spec}(\underline{A} / \pi \underline{A})$. If $\underline{A} / \pi \underline{A}$ is a domain, the residue norm $|\cdot|_{A}$ is multiplicative and, hence, defines a point in $M(A)$. This point specializes to the generic point of $\operatorname{Spec}(\underline{A} / \pi \underline{A})$, and it is the only point in $M(A)$ with this property.

Proof. Surjectivity of $\operatorname{sp}_{A}$ follows from [Grothendieck 1961a, 7.1.7]. If $\underline{A} / \pi \underline{A}$ is a domain, then $|\cdot|_{\underline{A}} \in M(\bar{A})$ clearly specializes to $\pi \underline{A}$. Moreover, the local ring $\underline{A}_{\pi} \underline{A}$ is then a discrete valuation ring, such that every character $\chi$ of a point $x \in M(A)$ specializing to the generic point of Spec $\underline{A} / \pi \underline{A}$ is equivalent to the character given by the natural homomorphism from $A$ to the fraction field of the $\pi$-adic completion of $\underline{A}_{\pi \underline{A}}$. It follows that $x$ equals $|\cdot|_{\underline{A}}$.

One can easily verify that when $\underline{A} / \pi \underline{A}$ is a domain, then $\left\{|\cdot|_{A}\right\}$ is the Shilov boundary of $M(A)$ [Kappen 2009, 1.2.5.12]; we will not use this fact in the following. Let us now discuss the example of the open unit disc $\operatorname{sSp}\left(R \llbracket S \rrbracket \otimes_{R} K\right)$ :

Example 4.2. The set $M\left(R \llbracket S \rrbracket \otimes_{R} K\right)$ is naturally identified with the closure of the Berkovich open unit disc within $M(K\langle S\rangle)$, which is obtained by adding the Gauss point.

Proof. To understand the continuous map $i: M\left(R \llbracket S \rrbracket \otimes_{R} K\right) \rightarrow M(K\langle S\rangle)$ induced by the natural isometry $K\langle S\rangle \hookrightarrow R \llbracket S \rrbracket \otimes_{R} K$, we distinguish the points in $M\left(R \llbracket S \rrbracket \otimes_{R} K\right)$ with respect to their specializations to the scheme Spec $k \llbracket S \rrbracket$. Applying Lemma 4.1 to $\underline{A}=R \llbracket S \rrbracket$, we see that the unique point above the generic point of Spec $k \llbracket S \rrbracket$ is the Gauss point $|\cdot|_{\text {Gauss }}$, which maps to the Gauss point in $M(K\langle S\rangle)$ via $i$. If $x \in M\left(R \llbracket S \rrbracket \otimes_{R} K\right)$ is a point specializing to the special point of Spec $k \llbracket S \rrbracket$, then for any character $\chi_{x}$ representing $x$, the induced $R$-homomorphism $\dot{\chi}_{x}: R \llbracket S \rrbracket \rightarrow \mathscr{K}$ is continuous for the $(\pi, S)$-adic topology on $R \llbracket S \rrbracket$ and the valuation topology on $\mathscr{K}$. In particular, $\chi_{x}$ is determined by the $\chi_{x}$ image of the variable $S$. We conclude that the map $i$ is injective and that it maps 
the complement of the Gauss point onto the Berkovich open unit disc. The image of $i$ is the continuous image of a compact set and, hence, compact. Since $M(K\langle S\rangle)$ is Hausdorff, it follows that the image of $i$ is closed in $M(K\langle S\rangle)$.

Remark 4.3. Given a complete nontrivially valued nonarchimedean field $K$ with valuation ring $R$, one may wonder whether the points of the rigid open unit disc over $K$ lie dense in $M\left(R \llbracket S \rrbracket \otimes_{R} K\right)$; this question is called the one-dimensional nonarchimedean Corona problem. It is yet unanswered; see the introduction of [Deninger 2010] for a brief survey including other versions of nonarchimedean Corona problems. If $K$ is discretely valued (which is the overall assumption in this paper), our discussion of Example 4.2 above shows that the Corona question has a positive answer: indeed, let $Z \subseteq M\left(R \llbracket S \rrbracket \otimes_{R} K\right)$ be the closure of the set of classical points; then the image of $Z$ under the natural map $i$ to the $K$-analytic space $M(K\langle S\rangle)$ is closed. Working locally on $M(K\langle S\rangle)$, we see that $i(Z)$ contains the Berkovich open unit disc and, hence, its closure. We have seen in Example 4.2 that $i$ is injective onto that closure; thus it follows that $Z=M\left(R \llbracket S \rrbracket \otimes_{R} K\right)$. The onedimensional nonarchimedean Corona problem is significantly more challenging when $K$ is not discretely valued: then the ring $R \llbracket S \rrbracket \otimes_{R} K$ is not noetherian, it has maximal ideals of infinite height [van der Put 1974, Corollary 4.9], and it contains functions with infinitely many zeros on the rigid open unit disc.

It is natural to ask whether one can associate a topological space to a uniformly rigid $K$-space such that, in the semiaffinoid case, one recovers the construction $\operatorname{sSp} A \mapsto M(A)$ which we described above. However, the formation of $M(A)$ does not behave well with respect to localization; see the following example. This is not surprising: the Banach $K$-algebra structure on $A$ restricts to the $\pi$-adic topology on an $R$-model of ff type $\underline{A}$ for $A$, and complete localization of $\underline{A}$ with respect to the $\pi$-adic topology does in general not agree with complete localization with respect to the topology defined by the Jacobson radical. Similarly, the extended specialization map $\operatorname{sp}_{A}$ maps onto the algebraization $\operatorname{Spec}(\underline{A} / \pi \underline{A})$ of the special fiber $\operatorname{Spf}(\underline{A} / \pi \underline{A})$ of $\operatorname{Spf} \underline{A}$ whose formation, again, does in general not commute with localization.

Example 4.4. If $\underline{A}=R\langle X, Y\rangle \llbracket Z \rrbracket /(X Y-Z)$, equipped with the Jacobson-adic topology, and if $\underline{B}=\underline{A}_{\{X-Y\}}$, then the induced map $M(B) \rightarrow M(A)$ is not injective.

Proof. Let us write $\mathfrak{X}:=\operatorname{Spf} \underline{A}$, and let $\mathfrak{X}_{0}:=\operatorname{Spec} k[X, Y] /(X Y)$ denote the smallest subscheme of definition of $\mathfrak{X}$. Since $\mathfrak{X}$ is formally smooth over $R$, its special fiber $\mathfrak{X}_{k}$ is formally smooth over $k$. The underlying topological space $\left|\mathfrak{X}_{k}\right|=$ $\left|\mathfrak{X}_{0}\right|$ is connected; hence the ring $\underline{A} / \pi \underline{A}$ is a domain. By Lemma 4.1, there exists a unique point $|\cdot|_{\underline{A}}$ of $M(A)$ specializing to the generic point of the algebraization $\mathfrak{X}_{k}^{\pi}:=\operatorname{Spec}(\underline{A} / \pi \underline{A})$ of the special fiber $\mathfrak{X}_{k}=\operatorname{Spf}(\underline{A} / \pi \underline{A})$ of $\mathfrak{X}$. On the other 
hand, let us consider the open formal subscheme $\mathfrak{U}:=\operatorname{Spf} \underline{B}$ of $\mathfrak{X}$. Its underlying smallest subscheme of definition $\mathfrak{U}_{0}$ is

$$
\mathfrak{U}_{0}=\operatorname{Spec}(k[X, Y] /(X Y))_{X-Y}=\operatorname{Spec}\left(k\left[X, X^{-1}\right]\right) \amalg \operatorname{Spec}\left(k\left[Y, Y^{-1}\right]\right),
$$

so $\mathfrak{U}$ has exactly two connected components. We conclude that $\underline{B}$ is a nontrivial direct sum $\underline{B}_{1} \oplus \underline{B}_{2}$ of flat $R$-algebras of ff type. Since $\mathfrak{U}$ is formally $R$ smooth, we see that $\underline{B}_{i} / \pi \underline{B}_{i}$ is a domain for $i=1,2$. We obtain an induced nontrivial decomposition $B=B_{1} \oplus B_{2}$ and, hence, a nontrivial decomposition $M(B)=M\left(B_{1}\right) \amalg M\left(B_{2}\right)$. By the proof of the statement in Example 4.2, there exist unique elements $|\cdot|_{B_{i}} \in M\left(B_{i}\right), i=1,2$, specializing to the respective generic point of $\mathfrak{U}_{i, k}^{\pi}:=\operatorname{Spec} \underline{B}_{i} / \pi \underline{B}_{i}$. To prove that the natural map $M(B) \rightarrow M(A)$ is not injective, it suffices to see that it maps the elements $|\cdot|_{\underline{B}_{1}},|\cdot|_{\underline{B}_{2}}$ in $M(B)$ to $|\cdot|_{\underline{A}}$. By functoriality of the specialization map, it thus suffices to observe that the natural morphism $\mathfrak{U}_{i, k}^{\pi} \rightarrow \mathfrak{X}_{k}^{\pi}$ maps the generic point to the generic point. However, this is clear because $\underline{A} / \pi \underline{A} \rightarrow \underline{B}_{i} / \pi \underline{B}_{i}$ is injective. Indeed, it is a flat homomorphism of domains, where flatness follows from the fact that $\mathfrak{U}_{i, k} \rightarrow \mathfrak{X}_{k}$ is an open immersion of formal schemes.

In the light of Example 4.4, it is unclear how to define a global analog of $M(A)$. Nonetheless, we think that a quasicompact uniformly rigid $K$-space $X$ should be viewed as a compactification of its underlying rigid $K$-space $X^{\mathrm{r}}$. This should be made more precise by studying the topos of $X$.

\section{Acknowledgements}

The present paper contains parts of the first chapter of the author's dissertation [Kappen 2009]. He would like to express his gratitude to his thesis advisor Siegfried Bosch. He also thanks Brian Conrad, Ofer Gabber, Ulrich Görtz, Philipp Hartwig, Simon Hüsken, Christian Wahle and the referee for helpful discussions and comments, and he would like to thank the Massachusetts Institute of Technology for its hospitality.

This work was financially supported by the Studienstiftung (German National Academic Foundation), by the Graduiertenkolleg Analytische Topologie und Metageometrie of the University of Münster and by the Hamburger Stiftung für Internationale Forschungs- und Studienvorhaben; the author would like to extend his gratitude to these institutions.

\section{References}

[Berkovich 1990] V. G. Berkovich, Spectral theory and analytic geometry over non-archimedean fields, Mathematical Surveys and Monographs 33, American Mathematical Society, Providence, RI, 1990. MR 91k:32038 Zbl 0715.14013 
[Berkovich 1996] V. G. Berkovich, "Vanishing cycles for formal schemes, II", Invent. Math. 125:2 (1996), 367-390. MR 98k:14031 Zbl 0852.14002

[Berthelot 1996] P. Berthelot, "Cohomologie rigide et cohomologie rigide à supports propres", preprint, Université de Rennes 1, 1996, Available at http://perso.univ-rennes1.fr/pierre.berthelot/publis/ Cohomologie_Rigide_I.p\% df.

[Bosch 2005] S. Bosch, "Lectures on formal and rigid geometry", preprint, Universität Münster, 2005, Available at http://www.math.uni-muenster.de/sfb/about/publ/heft378.pdf.

[Bosch and Lütkebohmert 1993a] S. Bosch and W. Lütkebohmert, "Formal and rigid geometry, I: Rigid spaces”, Math. Ann. 295:2 (1993), 291-317. MR 94a:11090 Zbl 0808.14017

[Bosch and Lütkebohmert 1993b] S. Bosch and W. Lütkebohmert, "Formal and rigid geometry, II: Flattening techniques", Math. Ann. 296:3 (1993), 403-429. MR 94e:11070 Zbl 0808.14018

[Bosch et al. 1984] S. Bosch, U. Güntzer, and R. Remmert, Non-Archimedean analysis: A systematic approach to rigid analytic geometry, Grundlehren der Mathematischen Wissenschaften 261, Springer, Berlin, 1984. MR 86b:32031 Zbl 0539.14017

[Bourbaki 1998] N. Bourbaki, Commutative algebra: Chapters 1-7, Elements of Mathematics, Springer, Berlin, 1998. MR 2001g:13001 Zbl 0902.13001

[Chai 2003] C.-L. Chai, "A bisection of the Artin conductor", preprint, University of Pennsylvania, 2003, Available at http://www.math.upenn.edu/ chai/papers_pdf/bAcond_v21.pdf.

[Conrad 1999] B. Conrad, "Irreducible components of rigid spaces", Ann. Inst. Fourier (Grenoble) 49:2 (1999), 473-541. MR 2001c:14045 Zbl 0928.32011

[Conrad 2006] B. Conrad, "Relative ampleness in rigid geometry", Ann. Inst. Fourier (Grenoble) 56:4 (2006), 1049-1126. MR 2007h:14029 Zbl 1125.14009

[Deninger 2010] C. Deninger, "Invariant functions on $p$-divisible groups and the $p$-adic corona problem”, Tokyo J. Math. 33:2 (2010), 393-406. MR 2779265 Zbl 05869972

[Eisenbud 1995] D. Eisenbud, Commutative algebra, with a view toward algebraic geometry, Graduate Texts in Mathematics 150, Springer, New York, 1995. MR 97a:13001 Zbl 0819.13001

[Grothendieck 1961a] A. Grothendieck, "Éléments de géométrie algébrique, II: Étude globale élémentaire de quelques classes de morphismes", Inst. Hautes Études Sci. Publ. Math. 8 (1961), 5-222. MR 36 \#177b Zbl 0118.36206

[Grothendieck 1961b] A. Grothendieck, "Éléments de géométrie algébrique, III: Étude cohomologique des faisceaux cohérents, I", Inst. Hautes Études Sci. Publ. Math. 11 (1961), 5-167. MR 36 \#177c Zbl 0118.36206

[Grothendieck 1964] A. Grothendieck, "Éléments de géométrie algébrique, IV: Étude locale des schémas et des morphismes de schémas, I", Inst. Hautes Études Sci. Publ. Math. 20 (1964), 5-259. MR 30 \#3885 Zbl 0136.15901

[Grothendieck and Dieudonné 1960] A. Grothendieck and J. Dieudonné, "Éléments de géométrie algébrique, I: Le langage des schémas", Inst. Hautes Études Sci. Publ. Math. 4 (1960), 1-228. MR 36 \#177a Zbl 0118.36206

[Grothendieck and Dieudonné 1971] A. Grothendieck and J. Dieudonné, Éléments de géométrie algébrique, I, Die Grundlehren der mathematischen Wissenschaften in Einzeldarstellungen 166, Springer, Berlin, 1971. Zbl 0203.23301

[Huber 1996] R. Huber, Étale cohomology of rigid analytic varieties and adic spaces, Aspects of Mathematics E30, Friedr. Vieweg, Braunschweig, 1996. MR 2001c:14046 Zbl 0868.14010

[Huber 2007] R. Huber, "A finiteness result for the compactly supported cohomology of rigid analytic varieties, II", Ann. Inst. Fourier (Grenoble) 57:3 (2007), 973-1017. MR 2008i:14037 Zbl 1146.14015 
[de Jong 1995] A. J. de Jong, "Crystalline Dieudonné module theory via formal and rigid geometry", Inst. Hautes Études Sci. Publ. Math. 82 (1995), 5-96. Erratum in 87 (1998), 175. MR 97f:14047 Zbl 0864.14009

[de Jong 1998] J. de Jong, "Erratum to: 'Crystalline Dieudonné module theory via formal and rigid geometry”, Inst. Hautes Études Sci. Publ. Math. 87 (1998), 175. MR 99i:14050

[Kappen 2009] C. Kappen, Uniformly rigid spaces and Néron models of formally finite type, Thesis, Universität Münster, 2009. Zbl 1219.14002 arXiv 1003.1022

[Kiehl 1967] R. Kiehl, "Theorem A und Theorem B in der nichtarchimedischen Funktionentheorie", Invent. Math. 2 (1967), 256-273. MR 35 \#1834 Zbl 0202.20201

[Lipshitz and Robinson 2000] L. Lipshitz and Z. Robinson, Rings of separated power series and quasi-affinoid geometry, Astérisque 264, Société Math. de France, Paris, 2000. MR 2001g:32017 Zbl 0957.32011

[Lütkebohmert 1990] W. Lütkebohmert, "Formal-algebraic and rigid-analytic geometry", Math. Ann. 286:1-3 (1990), 341-371. MR 90m:14015 Zbl 0716.32022

[Nicaise 2009] J. Nicaise, "A trace formula for rigid varieties, and motivic Weil generating series for formal schemes”, Math. Ann. 343:2 (2009), 285-349. MR 2010b:14043 Zbl 1177.14050

[van der Put 1974] M. van der Put, "The non-archimedean corona problem”, pp. 287-317 in Table ronde d'analyse non archimédienne (Paris, 1972), Bull. Soc. Math. France, Mém. 39-40, Soc. Math. France, Paris, 1974. Supplement to Bull. Soc. Math. France, Mém. 102. MR 51 \#939 Zbl 0303.46048

[Rapoport and Zink 1996] M. Rapoport and T. Zink, Period spaces for p-divisible groups, Annals of Mathematics Studies 141, Princeton University Press, 1996. MR 97f:14023 Zbl 0873.14039

[Raynaud 1974] M. Raynaud, “Géométrie analytique rigide d'après Tate, Kiehl, ...”, pp. 319-327 in Table ronde d'analyse non archimédienne (Paris, 1972), Bull. Soc. Math. France, Mém. 39-40, Soc. Math. France, Paris, 1974. Supplement to Bull. Soc. Math. France, Mém., 102. MR 57 \#10012 Zbl 0299.14003

[Strauch 2008] M. Strauch, "Deformation spaces of one-dimensional formal modules and their cohomology”, Adv. Math. 217:3 (2008), 889-951. MR 2009a:22014 Zbl 1140.22017

[Temkin 2008] M. Temkin, "Desingularization of quasi-excellent schemes in characteristic zero", Adv. Math. 219:2 (2008), 488-522. MR 2009h:14027 Zbl 1146.14009

[Valabrega 1975] P. Valabrega, "On the excellent property for power series rings over polynomial rings”, J. Math. Kyoto Univ. 15:2 (1975), 387-395. MR 51 \#12852 Zbl 0306.13011

[Valabrega 1976] P. Valabrega, "A few theorems on completion of excellent rings", Nagoya Math. J. 61 (1976), 127-133. MR 53 \#10790 Zbl 0319.13008

Communicated by Brian Conrad

Received 2010-09-06 Revised 2011-02-22 Accepted 2011-03-25

christian.kappen@uni-due.de Institut für Experimentelle Mathematik, Universität Duisburg-Essen, Ellernstrasse 29, D-45326 Essen, Germany http://esaga.uni-due.de/christian.kappen 


\section{Algebra \& Number Theory}

msp.berkeley.edu/ant

\section{EDITORS}

MANAGING EDITOR

Bjorn Poonen

Massachusetts Institute of Technology

Cambridge, USA

\author{
EDITORIAL BOARD CHAIR \\ David Eisenbud \\ University of California \\ Berkeley, USA
}

\section{BOARD OF EDITORS}

Georgia Benkart

Dave Benson

Richard E. Borcherds

John H. Coates

J-L. Colliot-Thélène

Brian D. Conrad

Hélène Esnault

Hubert Flenner

Edward Frenkel

Andrew Granville

Joseph Gubeladze

Ehud Hrushovski

Craig Huneke

Mikhail Kapranov

Yujiro Kawamata

János Kollár

Yuri Manin

Barry Mazur

Philippe Michel

Susan Montgomery
University of Wisconsin, Madison, USA

University of Aberdeen, Scotland

University of California, Berkeley, USA

University of Cambridge, UK

CNRS, Université Paris-Sud, France

University of Michigan, USA

Universität Duisburg-Essen, Germany

Ruhr-Universität, Germany

University of California, Berkeley, USA

Université de Montréal, Canada

San Francisco State University, USA

Hebrew University, Israel

University of Kansas, USA

Yale University, USA

University of Tokyo, Japan

Princeton University, USA

Northwestern University, USA

Harvard University, USA

École Polytechnique Fédérale de Lausanne

University of Southern California, USA
Shigefumi Mori

Raman Parimala

Jonathan Pila

Victor Reiner

Karl Rubin

Peter Sarnak

Joseph H. Silverman

Michael Singer

Ronald Solomon

Vasudevan Srinivas

J. Toby Stafford

Bernd Sturmfels

Richard Taylor

Ravi Vakil

Michel van den Bergh

Marie-France Vignéras

Kei-Ichi Watanabe

Andrei Zelevinsky

Efim Zelmanov
RIMS, Kyoto University, Japan

Emory University, USA

University of Oxford, UK

University of Minnesota, USA

University of California, Irvine, USA

Princeton University, USA

Brown University, USA

North Carolina State University, USA

Ohio State University, USA

Tata Inst. of Fund. Research, India

University of Michigan, USA

University of California, Berkeley, USA

Harvard University, USA

Stanford University, USA

Hasselt University, Belgium

Université Paris VII, France

Nihon University, Japan

Northeastern University, USA

University of California, San Diego, USA

\section{PRODUCTION}

contact@msp.org

Silvio Levy, Scientific Editor

See inside back cover or www.jant.org for submission instructions.

The subscription price for 2012 is US \$175/year for the electronic version, and \$275/year (+\$40 shipping outside the US) for print and electronic. Subscriptions, requests for back issues from the last three years and changes of subscribers address should be sent to Mathematical Sciences Publishers, Department of Mathematics, University of California, Berkeley, CA 94720-3840, USA.

Algebra \& Number Theory (ISSN 1937-0652) at Mathematical Sciences Publishers, Department of Mathematics, University of California, Berkeley, CA 94720-3840 is published continuously online. Periodical rate postage paid at Berkeley, CA 94704, and additional mailing offices.

ANT peer review and production are managed by EditFLOW ${ }^{\circledR}$ from Mathematical Sciences Publishers.

PUBLISHED BY

mathematical sciences publishers

http://msp.org/

A NON-PROFIT CORPORATION

Typeset in IAT $_{\mathrm{E}} \mathrm{X}$

Copyright (C2012 by Mathematical Sciences Publishers 


\section{Algebra \& Number Theory}

Volume $6 \quad$ No. $2 \quad 2012$

Arithmetic of singular Enriques surfaces

Klaus HuleK and MatThias SchÜtT

An upper bound on the Abbes-Saito filtration for finite flat group schemes and applications

YICHAO TIAN

On the smallest number of generators and the probability of generating an algebra

Rostyslav V. Kravchenko, Marcin Mazur and Bogdan V. Petrenko

Moving lemma for additive higher Chow groups

AMALENDU KRISHNA and JINHYUN PARK

CHRISTOPHER GOFF

Uniformly rigid spaces

CHRISTIAN KAPPEN

On a conjecture of Kontsevich and Soibelman

LÊ QUY THUONG 